The Astronomical Journal, 121:3235-3253, 2001 June

(C) 2001. The American Astronomical Society. All rights reserved. Printed in U.S.A.

\title{
BROWN DWARF COMPANIONS TO G-TYPE STARS. I. GLIESE 417B AND GLIESE 584C ${ }^{1}$
}

\author{
J. DAVY KiRKPATRICK \\ Infrared Processing and Analysis Center, MS 100-22, California Institute of Technology, Pasadena, CA 91125; davy@ipac.caltech.edu \\ Conard C. Dahn AND David G. Monet \\ US Naval Observatory, P.O. Box 1149, Flagstaff, AZ 86002; dahn@nofs.navy.mil, dgm@nofs.navy.mil
}

I. NEILL REID

Space Telescope Science Institute, 3700 San Martin Drive, Baltimore, MD 21218; inr@stsci.edu; also Department of Physics and Astronomy, University of Pennsylvania, Philadelphia, PA 19104-6396

JoHN E. Gizis

Infrared Processing and Analysis Center, MS 100-22, California Institute of Technology, Pasadena, CA 91125; gizis@ipac.caltech.edu

JAMES LIEBERT

Steward Observatory, University of Arizona, Tucson, AZ 85721; liebert@as.arizona.edu

AND

Adam J. Burgasser

Department of Physics, MS 103-33, California Institute of Technology, Pasadena, CA 91125; diver@its.caltech.edu Received 2000 December 26; accepted 2001 March 12

\begin{abstract}
We present astrometric and spectroscopic observations confirming that two nearby $\mathrm{G}$ dwarf systems (Gliese $417=\mathrm{BD}+36^{\circ} 2162$ and Gliese $584 \mathrm{AB}=\eta \mathrm{CrB} \mathrm{AB}$ ) have a widely separated, $\mathrm{L}$ dwarf, substellar companion. Using age estimates of the $\mathrm{G}$ dwarf primaries, we estimate masses for these $\mathrm{L}$ dwarfs from theoretical evolutionary tracks. For the L4.5 dwarf Gl 417B we estimate an age of 0.08-0.3 Gyr and a mass of $0.035 \pm 0.015 M_{\odot}$. For the L8 dwarf G1 584C we estimate an age of 1.0-2.5 Gyr and a mass of $0.060 \pm 0.015 \bar{M}_{\odot}$. This latter object also shows evidence of spectrum variability, which may be due to surface inhomogeneities rotating into and out of view. These new companions are also compared to six other L dwarf and $\mathrm{T}$ dwarf companions previously recognized. For the $\mathrm{L}$ dwarf companions, ages implied by the presence or absence of lithium are consistent with ages inferred from the primaries alone.

Key words: infrared: stars - stars: atmospheres — stars: distances — stars: fundamental parameters stars: low-mass, brown dwarfs
\end{abstract}

\section{INTRODUCTION}

The search for brown dwarfs and planets as close companions to nearby stars has met with spectacular success in recent years. Radial velocity surveys of F, G, K, and M stars have turned up many planetary companions with masses of $\sim 0.001 M_{\odot}$ and higher (see Marcy \& Butler 2000 and references therein). High spatial resolution space-based studies have also revealed low-mass stellar and substellar companions to nearby stars and brown dwarfs (HST NICMOSLowrance et al. 1999, 2000, Martín, Brandner, \& Basri 1999; HST WFPC2-Reid et al. 2001). Ground-based adaptive optics, coronagraphy, and small-field imaging have also resulted in several important discoveries (e.g., Nakajima et al. 1995; Rebolo et al. 1998).

Despite the recent successes, the search for similar companions at much wider separations has seen little advancement since the days of van Biesbroeck's $(1944,1961)$ photographic search for common-proper-motion companions to high motion stars. Finding companions at wide separations involves one main limitation: large pieces of sky

${ }^{1}$ Portions of the data presented herein were obtained at the W. M. Keck Observatory which is operated as a scientific partnership among the California Institute of Technology, the University of California, and the National Aeronautics and Space Administration. The Observatory was made possible by the generous financial support of the W. M. Keck Foundation. have to be canvassed. In the case of the triple star $\alpha$ Centauri $\mathrm{AB}+$ Proxima Centauri, the apparent separation is a staggering 2.18 degrees, which at the system's distance of 1.3 pc corresponds to a separation of $\sim 0.04 \mathrm{pc}$. Even wider physical separations are known, however, such as the $\gamma$ Ceti $\mathrm{AB}+\mathrm{Gl} 106.1 \mathrm{C}$ system with a separation of $\sim 0.09 \mathrm{pc}$.

The best available observational evidence suggests that widely separated companions exist out to distances of $\sim 0.1$ pc (Latham et al. 1984; Weinberg 1990). Companions at significantly wider separations are highly unlikely due to dynamical interactions with passing field stars (Retterer \& King 1982). Using data from Close, Richer, \& Crabtree (1990) it is found that $\sim 5 \%$ of systems (or $\sim 3 \%$ of stars) with $M_{V} \leq 9.0$ (spectral type $\sim \mathrm{M} 0 \mathrm{~V}$ ) have a companion wider than $0.01 \mathrm{pc}$. When $\mathrm{M}$ dwarfs are also included in the analysis, it is found that a similar number ( $\sim 6 \%$ of systems) have companions at these separations (Fischer \& Marcy 1992).

Estimates by Reid et al. (Reid 1999; Reid et al. 1999) show that there could be perhaps as many as two times the number of brown dwarfs as stars in the solar neighborhood. If the distribution of substellar companions mimics that of stellar companions we might expect up to $10 \%$ of disk stars to have a brown dwarf companion at a separation greater than $0.01 \mathrm{pc}$. However, the results of Marcy \& Butler (2000) show that there is a distinct difference between the frequency of stellar and substellar companions at very close separations. They note a paucity of brown dwarf 
companions - the "brown dwarf desert"-at separations less than $3 \mathrm{AU}$ around solar-type main-sequence stars; fewer than $0.5 \%$ of their stars have a brown dwarf companion at these separations. The reason for this difference remains unexplained, but a possible key is in finding whether a similar "brown dwarf desert" exists at wider separations from main-sequence stars.

Today's deep, large-area surveys like the Deep NearInfrared Survey (DENIS; Epchtein et al. 1997), the Two Micron All Sky Survey (2MASS; Skrutskie et al. 1997), and the Sloan Digital Sky Survey (SDSS; York et al. 2000) are presenting a wealth of new material with which lower luminosity companions to nearby stars can be found at very wide apparent separations. Two such companions with $\mathrm{K}$ dwarf primaries have already been found by 2MASS and have been announced elsewhere-Gl 570D (Burgasser et al. 2000) and GJ 1048B (Gizis et al. 2000). Here we discuss two additional brown dwarfs, Gl 417B and Gl 584C, both of which are $\mathrm{L}$ dwarf companions to nearby $\mathrm{G}$ dwarfs.

The discovery of these L dwarfs is described in $\S 2$. Details on the spectroscopic follow-up are given in $\S 3$ along with astrometric measurements showing that the two $\mathrm{L}$ dwarfs share common proper motion with the nearby $G$ stars. Using extensive supplementary observations of the $G$ dwarf primaries, we derive age estimates for the systems and mass estimates for the L dwarf companions in $\S 4$. We compare these two brown dwarfs to other known companions in $\S 5$ and summarize our conclusions in $\S 6$.

\section{DISCOVERY}

While searching the 2MASS data for late-L dwarf suspects (Kirkpatrick et al. 2000), we uncovered two candidates lying in close proximity to known nearby stars. The first candidate has a designation of 2MASSI $\mathrm{J} 1112256+354813$ and is shown in the finder chart of Figure 1 (left panels). As an L-dwarf candidate it is bright and quite red: $K_{s}=12.69 \pm 0.05$ and $J-K_{s}=1.88 \pm 0.06$. As shown in the figure it lies $90^{\prime \prime}$ southwest of the nearby G

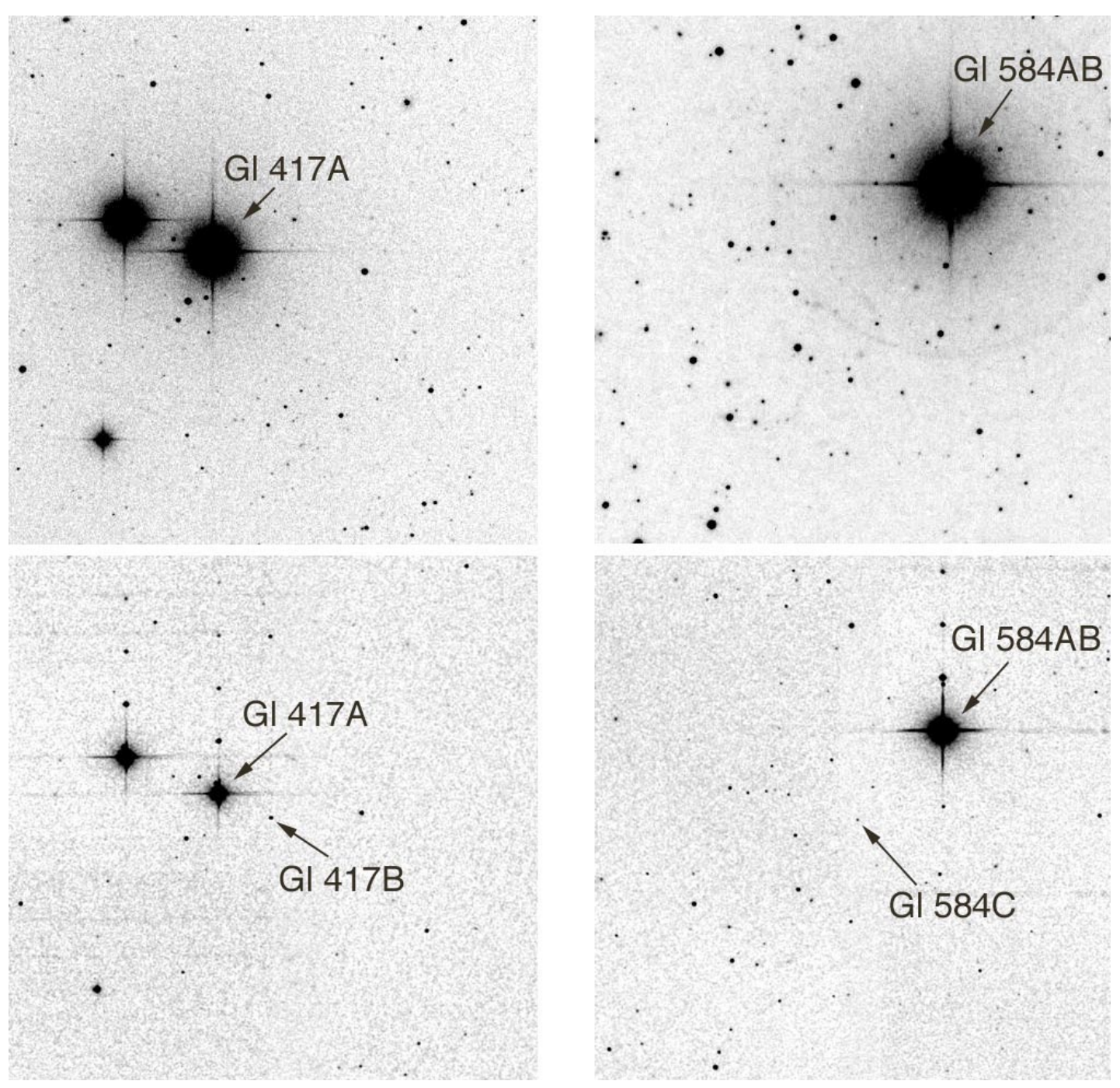

Fig. 1.-Finder charts for both systems. All four images are 13'7 on a side with north up and east to the left. Left panels: The top image shows the 1953-epoch POSS-I $E$-band image for G1 417, and the bottom image shows the 1998-epoch 2MASS $K_{s}$ image. The L dwarf companion, Gl 417B, is visible only in the near-infrared view. Right panels: The top image shows the 1954-epoch POSS-I E-band image for G1 584, and the bottom image shows the 2000-epoch 2MASS $K_{s}$ image. The L dwarf companion, G1 584C, is visible only in the near-infrared view. 
dwarf G1 417 (aka HR 4345, HD 97334, BD+36 2162). ${ }^{2}$ Hereafter we will refer to the primary as G1 $417 \mathrm{~A}$ and the L dwarf candidate as G1 417B.

The second candidate has a designation of 2MASSW $\mathrm{J} 1523226+301456$ and is shown in the finder chart of Figure 1 (right panels). It is even redder than the previous candidate $-J-K_{s}=2.09 \pm 0.13 \quad$ with $\quad K_{s}=14.24$ \pm 0.07 -and lies $194^{\prime \prime}$ southeast of the nearby G dwarf double Gl 584AB (aka $\eta$ CrB AB, HR 5727/8, HD 137107/8, $\mathrm{BD}+30^{\circ} 2653$, ADS $\left.9617 \mathrm{AB}\right){ }^{3}{ }^{3}$ Hereafter we will refer to the L dwarf candidate as G1 584C.

As Figure 1 shows, neither of these $\mathrm{L}$ dwarf candidates is visible on the POSS-I E (red) plates, indicating very red optical-minus-infrared colors. The sources are also invisible in on-line images of the POSS-II J (blue) and F (red) plates. Thus, the 2MASS images represent the first-epoch detections for both candidates.

\section{FOLLOW-UP OBSERVATIONS}

\subsection{Astrometric Confirmation}

The $G$ dwarf primaries have small motions on the sky $-0.29 \mathrm{yr}^{-1}$ for Gl 417A and $0.22 \mathrm{yr}^{-1}$ for Gl 584C (Perryman et al. 1997) - so moderately precise astrometry is needed for speedy verification of common proper motion between the $\mathrm{G}$ dwarf primaries and the L dwarf companions. Toward this end, both systems have been observed at multiple epochs starting in 1999 May at the US Naval Observatory in Flagstaff, Arizona. Both Gliese systems were observed with the 61 inch Strand Astrometric Reflector using the ND9 camera. This camera employs a $3 \mathrm{~mm}$ $\left(\sim 40^{\prime \prime}\right)$ diameter attenuating spot deposited upon an opti-

\footnotetext{
${ }^{2}$ The field around G1 417A contains several relatively bright stars. BD $+36^{\circ} 2164(V=7.2)$ lies $\sim 156^{\prime \prime}$ to the northeast of G1 417A $(V=6.4)$. The Hipparcos parallax and proper motion measurements (Perryman et al. 1997) for this star show that it is unassociated with Gl 417A. A dimmer star $(V=12.3)$ referred to as $\mathrm{BD}+36^{\circ} 2162 \mathrm{C}$ is located $\sim 86^{\prime \prime}$ southeast of Gl 417A. This star has no Hipparcos measures, but it is also unassociated because a comparison of the DSS and XDSS images shows that it exhibits no detectable proper motion (whereas that of Gl 417A is obvious). Another bright star, $\mathrm{BD}+36^{\circ} 2165$ (LHS $2373 ; V=9.8$ ), is located $\sim 312^{\prime \prime}$ southeast of Gl 417A. This star exhibits motion that is larger and along a substantially different position angle than that of Gl 417A, and Hipparcos also finds a much greater distance. Hence, this object is also not associated.

${ }^{3}$ Two other relatively bright stars are also listed in various catalogs as members of this system. A star known as BD $+30^{\circ} 2653 \mathrm{C}$ (ADS 9617C, $V=13.4$ ) is located 72" due north of G1 584AB. Another star, ADS 9617D $(V=11.0)$ is located $217^{\prime \prime}$ to the northeast of G1 584AB. A comparison of the 1954-epoch POSS-I images to the 2000-epoch 2MASS images shows that neither of these stars shares the proper motion of Gl 584AB, so neither is physically associated.
}

cally flat substrate that is mounted $\sim 1 \mathrm{~mm}$ in front of the Tek2K CCD. This spot is located at the center of the $\sim 10^{\prime} \times 10^{\prime}$ field of view and provides approximately 9.0 mag of compensation. Images were centered with $G$ dwarf primaries behind the spot so that both the bright primaries and L dwarf candidate secondaries could be recorded simultaneously. An astrometrically flat, wide- $I$ interference filter $\left(\lambda_{c} \sim 8100 \AA\right.$; FWHM $\left.\sim 1910 \AA\right)$ was employed for the observations.

The astrometric observations are summarized in Table 1, which gives the epochs of the observations, number of images taken, parallax factors in right ascension and declination, and the mean components of the projected tangents of the zenith distances for each set of observations. The parallax factors represent the effective triangulation baseline (in AU) at the time of observation, and the sizable, nonmonotonic variation in parallax factor over the spread of epochs assures good separation of parallax from proper motion. The components of the projected tangent of the zenith distance are relevant to potential contamination of the measures by differential color refraction (DCR; cf. Monet et al. 1992) which will be addressed below.

For the Gl 417 system, relative parallaxes and proper motions could be measured for both the A and B components using 8-10 minute integrations. A reference frame of 16 stars, well distributed around both target objects, was initially employed for the solution. Table $2 \mathrm{~A}$ summarizes the astrometric results for G1 417, with the first line presenting the Hipparcos (Perryman et al. 1997) results for the A component for comparison. The results for both A and B components from the full USNO solution (13 exposures, 16 reference stars) are given in the second and third lines.

In comparing the USNO parallax and proper motion results with the Hipparcos values, it is important to remember that the USNO determinations are made from narrowfield, differential measures and are, therefore, strictly "relative" to the mean parallaxes and mean proper motions of the reference stars which are both set identically equal to zero by the formal least-squares reduction algorithms. Relative parallaxes can be "corrected" to absolute by estimating the mean parallax of the reference stars employed, either from a galactic model, or better from (spectro)photometric observations of the individual stars. The Hipparcos results, on the other hand, are derived from global solutions to whole-sky observations of many thousands of stars and should, therefore, be truly "absolute." As discussed below, photometric observations for faint stars in the near proximity of such bright primary stars was not generally successful. However, based on other USNO parallax determinations for fields at similar Galactic latitudes as Gl $417\left(+67.3^{\circ}\right)$

TABLE 1

JOURNAL OF USNO ASTROMETRIC OBSERVATIONS

\begin{tabular}{ccccccc}
\hline \hline $\begin{array}{c}\text { Name of Object } \\
(1)\end{array}$ & $\begin{array}{c}\text { Obs. Epoch (yr) } \\
(2)\end{array}$ & $\begin{array}{c}\text { Number of Obs. } \\
(3)\end{array}$ & $\begin{array}{c}P_{\text {R.A. }} \\
(4)\end{array}$ & $\begin{array}{c}P_{\text {decl. }} \\
(5)\end{array}$ & $\begin{array}{c}\left\langle Z_{\text {R.A. }}\right\rangle \\
(6)\end{array}$ & $\begin{array}{c}\left\langle Z_{\text {decl. }}\right\rangle \\
(7)\end{array}$ \\
\hline G1 417B $\ldots \ldots . .$. & 1999.38 & 4 & -0.878 & +0.491 & +0.301 & +0.017 \\
& 2000.10 & 4 & +0.475 & +0.257 & -0.117 & +0.010 \\
& 2000.35 & 5 & -0.793 & +0.556 & -0.090 & +0.011 \\
& & & & & & \\
G1 584C $\ldots \ldots . .$. & 1999.38 & 3 & -0.072 & +0.773 & +0.243 & +0.104 \\
& 2000.34 & 1 & +0.128 & +0.731 & +0.055 & +0.084 \\
& 2000.51 & 1 & -0.751 & +0.624 & +0.073 & +0.084 \\
\hline
\end{tabular}


TABLE 2A

COMPARATIVE ASTROMETRY FOR Gl 417

\begin{tabular}{|c|c|c|c|c|}
\hline $\begin{array}{c}\text { Component } \\
\text { (1) }\end{array}$ & $\begin{array}{l}\pi \text { (mas) } \\
(2)\end{array}$ & $\underset{(3)}{\mu\left(\operatorname{mas} \mathrm{yr}^{-1}\right)}$ & $\begin{array}{l}\theta(\mathrm{deg}) \\
(4)\end{array}$ & $\begin{array}{l}\text { Source and Comments } \\
\text { (5) }\end{array}$ \\
\hline Gl 417A...... & $46.0 \pm 0.9$ & $291.0 \pm 01.0$ & $238.7 \pm 0.1$ & Hipparcos \\
\hline G1 417A...... & $44.3 \pm 1.5$ & $286.8 \pm 04.4$ & $239.8 \pm 1.4$ & USNO; no DCR correction; \\
\hline G1 417B ...... & $41.0 \pm 8.9$ & $277.3 \pm 13.2$ & $235.7 \pm 2.6$ & 13 exp.; 16 ref. stars \\
\hline Gl 417A...... & $45.2 \pm 1.2$ & $287.2 \pm 04.3$ & $240.3 \pm 1.4$ & USNO; no DCR correction; \\
\hline G1 417B ...... & $48.1 \pm 5.7$ & $292.0 \pm 08.9$ & $236.8 \pm 1.8$ & 12 exp.; 16 ref. stars \\
\hline G1 417A... & $43.9 \pm 1.2$ & $285.2 \pm 04.4$ & $240.8 \pm 1.5$ & USNO; no DCR correction; \\
\hline G1 417B ...... & $46.3 \pm 5.5$ & $291.6 \pm 08.9$ & $236.5 \pm 1.9$ & 12 exp.; 10 ref. stars \\
\hline Gl 417A.. & $44.5 \pm 1.5$ & $286.1 \pm 04.4$ & $239.7 \pm 1.4$ & USNO; 13 exp.; 16 ref. stars; \\
\hline Gl 417B ...... & $40.7 \pm 8.6$ & $287.5 \pm 12.6$ & $237.1 \pm 2.5$ & DCR with $V-I=1.0$ for ref stars \\
\hline G1 417A...... & $45.0 \pm 1.6$ & $284.4 \pm 04.4$ & $239.5 \pm 1.4$ & USNO; 13 exp.; 16 ref. stars; \\
\hline G1 417B ...... & $39.0 \pm 8.5$ & $285.8 \pm 12.7$ & $236.9 \pm 2.5$ & DCR with $V-I=1.7$ for ref. stars \\
\hline
\end{tabular}

and where similarly faint reference stars were observed individually with $V, V-I$ photometry, we can estimate a correction from relative to absolute parallax of approximately $0.9 \pm 0.2$ milliarcsec (mas) for the USNO determinations. In making comparisons between the USNO and Hipparcos parallax values, this amount should be added to the USNO relative parallaxes presented in Table 2A. When this is done, the agreement between the USNO and Hipparcos parallax results are very satisfactory and justify adopting the better determined Hipparcos astrometry for further discussions of the G1 417 system.

Alternate, equally viable, solutions employing various subsets of the full USNO data have been investigated. For example, one of the observations at epoch 2000.35 is noticeably weak, and the residuals for it stand out as $>2 \sigma$ outliers. If that observation is rejected, the results presented in the fourth and fifth lines of Table $2 \mathrm{~A}$ are obtained. In addition, six of the 16 reference stars employed in the previous solutions are either quite weak on several frames or fall in the region of the field affected by reflected light off of the compensation spot. If we further remove them from the astrometric solution, the results presented on the sixth and seventh lines are obtained. These are just example results from an array of solutions carried out for various combinations of exposure frames and reference stars. They are presented to demonstrate that, although the observational data is admittedly limited, the solutions they yield are indeed quite robust and do support the physical relationship between the $G$ dwarf primary and the $L$ dwarf secondary - that is, that the two objects are at the same distance and share a common proper motion.

The solutions presented thus far have not included any correction for differential color refraction (DCR). The wide- $I$ filter employed for the observations has been in routine use on the USNO parallax program since 1992 and a DCR correction for it has been calibrated using the method described in $\S 3.2$ of Monet et al. (1992). The resulting relation is essentially the same as shown in Figure 4 of that paper for the STWIDER bandpass but with an amplitude only 0.252 times as large. As in the case of the STWIDER calibration, the wide- $I$ calibration has only been measured for stars as red as late $\mathrm{M}$ dwarfs.
Extrapolation of this calibration to redder L dwarfsespecially to late L dwarfs, where the strong, broad KI absorption alters the energy distribution within the wide- $I$ bandpass significantly - is at best valid for observations confined to small range of hour angles. As the data in Table 1 show, the astrometric exposures presented here were mostly centered within 45 minutes of the meridian and there is only a small correlation between projected tangent of the zenith distance and parallax factor. Hence, we anticipate that the effects of DCR on the astrometry presented should not be too large.

Due to observational constraints, only rough estimates of the DCR effects are possible. Measures of $V-I$ colors for both the L dwarfs and the astrometric reference stars were not possible using USNO telescopes and instrumentation. The L dwarfs are simply too faint at $V$ band and even attempts to observe them at $R$ band were unsuccessful. Even the reference stars were, in most cases, unobservable due to the larger amounts of scattered light from the $G$ dwarf primaries at $V$ band. Hence, estimated colors have been adopted to illustrate the potential effect that DCR might produce.

Examination of reference star photometry for 23 USNO fields at similarly high Galactic latitudes shows that the majority of reference stars have $V-I$ colors between 0.8 and 2.5. Only an occasional star might be as blue as 0.6 or as red as 2.8. For six fields employing faint reference stars, the median $V-I$ colors of those stars ranged from 1.13 to 1.68. Therefore, solutions including DCR corrections were run assuming that all reference stars employed for the Gl 417 field had either $V-I=1.0$ or $V-I=1.7$. For G1 417A the measured value of $V-I=0.67$ was adopted from Hipparcos. For Gl 417B, a value of $V-I=6.2$ was assumed based on the measured value of $V-I=6.2 \pm 0.5$ obtained for 2MASSW $1507476-162738$ which is bright enough to be measured at $V$ band and which has a similar spectral type (L5) as Gl 417B (L4.5).

The astrometric solutions including these DCR estimates are given on the eighth through eleventh lines of Table $2 \mathrm{~A}$. Small but significant changes in the formal numbers are seen - especially for the L dwarf component - when these results are compared with the solutions neglecting DCR. 
However, the Table 2A results - which include just a few examples from an array of solutions carried out for various combinations of exposure frames, reference stars, and DCR estimates - demonstrate that, while the formal numbers can change somewhat (as expected), in all cases the solutions support a physical relationship between the $G$ dwarf primary (Gl 417A) and the L dwarf secondary (Gl 417B). Hence, we feel well justified in adopting the better determined Hipparcos astrometry in further discussions of the Gl 417 system.

The observational situation for the G1 584 system was much more difficult. The primary pair of $G$ dwarfs, Gl $584 \mathrm{AB}$, are recorded by the 61 inch telescope as partially resolved; that is, not sufficiently separated for astrometric measure of individual components and not sufficiently blended to permit reliable astrometry of the combined light. Furthermore, even when the $\mathrm{AB}$ pair was exposed to saturation behind the 9.0 mag compensation spot, the $L$ dwarf candidate companion was not recorded due to its extreme faintness at $I$ band. Following several trial exposures with the AB pair moved to the edge of the CCD field or even off the $C C D$, it was decided to leave the field positioned with the $A B$ pair behind the spot since this arrangement seemed to reduce the glare of scattered light from the $\mathrm{AB}$ pair into the region of the L dwarf. Exposure times of 20, 30, and 40 minutes were used at epoch 1999.38, and the results have relatively low $\mathrm{S} / \mathrm{N}$. Single 90 minute exposures were used at epochs 2000.34 and 2000.51 and gave better results.

Due to the increased amounts of scattered light from longer exposure times, the faintness of $\mathrm{Gl} 584 \mathrm{C}$, and the location of the target well into one quadrant of the usable field, the results for G1 584C are much less definitive. Only seven reference stars were usable, and three of these are very faint. With only a limited number of exposures and a poor reference frame, alternate solutions were not possible or gave very unsatisfactory results. Table $2 \mathrm{~B}$ gives the Hipparcos solution for G1 584AB along with USNO solutions first without a DCR correction and then with DCR estimates assuming a very uncertain $V-I=6.5$ for G1 584C. The agreement of the non-DCR USNO parallax solution with the Hipparcos value appears to be highly fortuitous, since the two DCR solutions show little agreement with the non-DCR solution. However, the proper motions from all three USNO solutions agree well enough with the Hipparcos results that it is very likely that $\mathrm{Gl} 584 \mathrm{C}$ has a physical relationship with Gl $584 \mathrm{AB}$ and is at the same distance. For further discussion we assume this to be the case and adopt the Hipparcos astrometry for the Gl 584 system.

\subsection{Spectroscopic Confirmation}

G1 417B and G1 584C were observed in 1998 December and 1999 March using the Low Resolution Imaging Spectrograph (LRIS; Oke et al. 1995) at the $10 \mathrm{~m} \mathrm{W.} \mathrm{M.}$ Keck Observatory on Mauna Kea, Hawaii. Table 3 contains a $\log$ of the observations. ${ }^{4}$ A $400 \mathrm{~g} \mathrm{~mm}^{-1}$ grating blazed at $8500 \AA$ was used with a 1" slit and $2048 \times 2048$ CCD to produce $9 \AA$ resolution spectra covering the range 6300-10100 $\AA$. The OG570 order-blocking filter was used to eliminate second-order light. The data were reduced and calibrated using standard IRAF routines. A $1 \mathrm{~s}$ dark exposure was used to remove the bias, and quartz-lamp flat-field exposures were used to normalize the response of the detector. The individual stellar spectra were extracted and sky subtracted using the "apextract" routine in IRAF, allowing for the slight curvature of a point-source spectrum viewed through the LRIS optics and using a template where necessary. Wavelength calibration was achieved using neon + argon arc lamp exposures taken after each program object. Finally, the spectra were flux-calibrated using observations of standards LTT 9491, Hiltner 600, LTT 1020, and Feige 56 from Hamuy et al. (1994). As these data were taken as part of our general spectral classification campaign, no data were acquired for telluric absorption correction. Hence, the Earth's atmospheric $\mathrm{O}_{2}$ bands at 6867-7000, 7594-7685 $\AA$ and $\mathrm{H}_{2} \mathrm{O}$ bands at 7186-7273, 8161-8282, $\sim 8950-9300, \sim 9300-9650 \AA$ are still present in the spectra. For all observations the slit was oriented at the parallactic angle to eliminate problems associated with differential refraction.

As shown in Figure 2, the spectra of both objects confirm that these are $\mathrm{L}$ dwarfs. The classifications for these two objects, which are fully discussed in Kirkpatrick et al. (2000), are L4.5 V for G1 417B and L8 V for G1 584C. Both objects exhibit Li I absorption at $6708 \AA$, as illustrated in the insets in Figure 2. The Li I absorption has an equivalent width of $11.5 \AA$ for Gl 417B and $7.4 \AA$ for Gl $584 \mathrm{C}$, though the spectrum of G1 $584 \mathrm{C}$ is much noisier in this wavelength regime. The presence of lithium absorption indicates that these two L dwarfs are substellar (Rebolo, Martín, \& Magazzù 1992).

\subsection{Spectrum Variability in $\mathrm{Gl} 584 \mathrm{C}$}

A comparison of the 1998 December 24 and 25 spectra of G1 $584 \mathrm{C}$ shows evidence for variability in some of the

\footnotetext{
${ }^{4}$ The other $\mathrm{L}$ dwarfs listed in this table are discussed in $\S 4.2$.
}

TABLE 2B

COMPARATIVE ASTROMETRY FOR G1 584

\begin{tabular}{|c|c|c|c|c|}
\hline $\begin{array}{l}\text { Component } \\
\text { (1) }\end{array}$ & $\begin{array}{l}\pi \text { (mas) } \\
(2)\end{array}$ & $\mu\left({\left.\operatorname{mas~} y r^{-1}\right)}_{(3)}\right.$ & $\begin{array}{l}\theta(\mathrm{deg}) \\
\quad(4)\end{array}$ & $\begin{array}{l}\text { Source and Comments } \\
\text { (5) }\end{array}$ \\
\hline G1 584AB ...... & $53.7 \pm 1.2$ & $216.7 \pm 01.7$ & $144.5 \pm 0.1$ & Hipparcos \\
\hline G1 $584 C \ldots \ldots$ & $51.7 \pm 5.4$ & $219.0 \pm 03.0$ & $132.5 \pm 1.0$ & $\begin{array}{l}\text { USNO; no DCR correction; } \\
5 \text { exp.; } 7 \text { ref. stars }\end{array}$ \\
\hline G1 584C ....... & $35.7 \pm 1.7$ & $214.9 \pm 01.9$ & $133.5 \pm 1.0$ & $\begin{array}{l}\text { USNO; } 5 \text { exp.; } 7 \text { ref. stars; } \\
\text { DCR with } V-I=1.0 \text { for ref. stars }\end{array}$ \\
\hline G1 584C ....... & $39.4 \pm 2.2$ & $215.6 \pm 02.0$ & $133.3 \pm 1.1$ & $\begin{array}{l}\text { USNO; } 5 \text { exp.; } 7 \text { ref. stars; } \\
\text { DCR with } V-I=1.7 \text { for ref. stars }\end{array}$ \\
\hline
\end{tabular}


TABLE 3

JOURNAL OF LRIS OBSERVATIONS FOR L DWARF COMPANIONS

\begin{tabular}{|c|c|c|c|}
\hline $\begin{array}{l}\text { Name of L Dwarf } \\
\text { (1) }\end{array}$ & $\begin{array}{l}\text { 2MASS Designation } \\
\text { (2) }\end{array}$ & $\begin{array}{l}\text { Obs. Date (UT) } \\
\text { (3) }\end{array}$ & $\begin{array}{c}\text { Exposure Time } \\
\text { (s) } \\
\text { (4) }\end{array}$ \\
\hline Gl 417B ........... & 2MASSI J1112256+354813 ${ }^{\mathrm{a}}$ & $\begin{array}{l}1998 \text { Dec } 14 \\
1999 \text { Mar } 04 \\
1999 \text { Mar } 05\end{array}$ & $\begin{array}{l}1200 \\
1200 \\
3600\end{array}$ \\
\hline G1 $584 \mathrm{C} \ldots \ldots \ldots \ldots$ & 2MASSW J1523226+301456 & $\begin{array}{l}1998 \text { Dec } 24 \\
1998 \text { Dec } 25 \\
1999 \text { Mar } 04 \\
1999 \text { Mar } 31\end{array}$ & $\begin{array}{l}3600 \\
4600 \\
3600 \\
4800\end{array}$ \\
\hline G 196-3B ......... & 2MASSW J1004207 + 502300 & $\begin{array}{l}1999 \text { Mar } 04 \\
1999 \text { Mar } 05\end{array}$ & $\begin{array}{l}2400 \\
3600\end{array}$ \\
\hline GJ 1001B ......... & 2MASSW J0004348-404405 & 1999 Jul 17 & 1200 \\
\hline GJ 1048B ......... & 2MASSI J0235599-233120 & 2000 Aug 23 & 844 \\
\hline
\end{tabular}

a Also known as 2MASSW J1112257 + 354813 (Kirkpatrick et al. 2000).

feature strengths, prompting us to reobserve it again on 1999 March 04 and 31. A more detailed observing log for G1 $584 \mathrm{C}$ is given in Table 4. As shown in the table, each night consisted of three or four back-to-back 20 minute integrations except for the last observation on 1998 December 25, which was cut short by advancing twilight.

Each of these individual exposures is shown in Figure 3 except for those of 1999 March 04, a night riddled by variable seeing and possibly light cirrus. There is excellent agreement in the overall spectral shape of the 11 individual integrations, but subtle differences are also seen.

Figure $4 a$ shows details of the 11 spectra over the 8350 $9050 \AA$ region. There is no appreciable telluric absorption in this wavelength range, but the telluric emission, as shown in Figure $4 b$, is comprised of myriad $\mathrm{OH}$ and $\mathrm{O}_{2}$ lines, all of which are blended at this resolution. Despite the strong sky lines, this telluric emission is easily subtracted from the $\mathrm{L}$ dwarf spectra, leaving only slight residuals at the $1 \%$ level. Individual spectra on 1998 December 25, for example, show excellent agreement despite the fact that the $\mathrm{O}_{2}$ and $\mathrm{OH}$ emission is greatly enhanced in the first, high-air mass spectra relative to the later ones. On any given night the shape of the spectrum in this region repeats well, but discrepancies in the band strength near $8650 \AA$ exist from one night to the next. Based on this figure we argue that this effect cannot be a consequence of poor sky subtraction. We therefore conclude that the variability see in Figure $4 a$ is inherent to the L dwarf itself.

Figure 5 shows details of the same spectra but in the 9050-9750 A region. Again, individual spectra for a particular night are internally self-consistent, but comparisons between nights reveal discrepancies. In this case, the discrepancies are found in the 9250-9600 $\AA \mathrm{H}_{2} \mathrm{O}$ absorption band, which has (as stated in $\S 3.2$ ) not been corrected for

TABLE 4

DetaIled Journal of KeCK LRIS ObSERVATIONS OF G1 584C

\begin{tabular}{|c|c|c|c|c|c|c|}
\hline $\begin{array}{l}\text { Observation Date (UT) } \\
\text { (1) }\end{array}$ & $\begin{array}{l}\text { UT at Start } \\
\text { (2) }\end{array}$ & $\begin{array}{c}\text { Air Mass at Start } \\
\text { (3) }\end{array}$ & $\begin{array}{l}\text { Exposure Time } \\
\text { (s) } \\
\text { (4) }\end{array}$ & $\begin{array}{c}\text { Seeing } \\
(\operatorname{arcsec}) \\
(5)\end{array}$ & $\begin{array}{l}\text { Moon } \\
(6)\end{array}$ & $\begin{array}{l}\text { Sky } \\
(7)\end{array}$ \\
\hline \multirow[t]{3}{*}{$1998 \operatorname{Dec} 24 \ldots \ldots \ldots \ldots$} & $14: 37$ & 2.52 & 1200 & 1.0 & \multirow[t]{3}{*}{ Below horizon } & \multirow[t]{3}{*}{ Clear } \\
\hline & $14: 58$ & 2.14 & 1200 & 1.0 & & \\
\hline & $15: 21$ & 1.84 & 1200 & 1.0 & & \\
\hline \multirow[t]{4}{*}{1998 Dec $25 \ldots \ldots \ldots \ldots$} & $14: 43$ & 2.33 & 1200 & 1.1 & \multirow[t]{4}{*}{ Below horizon } & \multirow[t]{4}{*}{ Clear } \\
\hline & $15: 03$ & 2.00 & 1200 & 1.2 & & \\
\hline & $15: 25$ & 1.77 & 1200 & 1.4 & & \\
\hline & $15: 46$ & 1.59 & 1000 & 1.4 & & \\
\hline \multirow[t]{3}{*}{1999 Mar $04 \ldots \ldots \ldots \ldots$} & $14: 01$ & 1.04 & 1200 & 0.8 & \multirow{3}{*}{$\begin{array}{c}95 \% \text { illum., } \\
49^{\circ} \text { away }\end{array}$} & \multirow[t]{3}{*}{ Lt. cirrus? } \\
\hline & $14: 29$ & 1.02 & 1200 & 1.1 & & \\
\hline & $14: 50$ & 1.01 & 1200 & 1.5 & & \\
\hline \multirow[t]{4}{*}{1999 Mar $31^{a} \ldots \ldots \ldots \ldots$} & $14: 02$ & 1.04 & 1200 & 0.6 & \multirow{4}{*}{$\begin{array}{c}100 \% \text { illum., } \\
50^{\circ} \text { away }\end{array}$} & \multirow[t]{4}{*}{ Clear } \\
\hline & $14: 23$ & 1.06 & 1200 & 0.6 & & \\
\hline & $14: 44$ & 1.09 & 1200 & 0.6 & & \\
\hline & $15: 05$ & 1.13 & 1200 & 0.6 & & \\
\hline
\end{tabular}

${ }^{\text {a }}$ This was a night of service observing. 




Fig. 2.- Spectra of the L dwarfs. (a) A $6000 \mathrm{~s}$ sum of the Gl 417B observations listed in Table 3. Shown in the inset is a detailed view of the spectrum between 6600 and $6800 \AA$ to highlight the lithium absorption at $6708 \AA$. (b) A 13,000 s sum of the 1998 December 24, 1998 December 25 , and 1999 March 31 observations listed in Table 3. The inset shows a detailed view near the lithium absorption line at $6708 \AA$, but here only the $8200 \mathrm{~s} \mathrm{sum}$ of the $1998 \mathrm{December}$ data is shown as those data have the best signal-to-noise in this region of the spectrum. In both the full panels and the insets, spectra have been normalized to one at $8250 \AA$ A. Prominent spectral features have been labeled in panel $(a)$.

telluric $\mathrm{H}_{2} \mathrm{O}$ absorption at the same wavelengths. Our experience at Keck on other clear runs where telluric absorption corrections were applied in this wavelength regime indicates that the depths of these telluric $\mathrm{H}_{2} \mathrm{O}$ bands can vary by at least $10 \%$ from one clear night to the next. The change seen here between the 1998 December 24 and 25 spectra is $\sim 15 \%$. Hence, the changes seen in the $9250-9600 \AA$ region may simply be due to variations in telluric $\mathrm{H}_{2} \mathrm{O}$ absorption. A rigorous monitoring program specifically designed to correct for telluric absorption would be able to determine whether or not a portion of the $\mathrm{H}_{2} \mathrm{O}$ variations seen here is intrinsic to the $\mathrm{L}$ dwarf itself.
Figure $6 a$ shows comparisons of the summed spectra for each night. Overall, the spectral slope repeats well from night to night as do the strengths of the Cs I lines. Pairwise overplots in Figures $6 b-6 f$ demonstrate the nightly differences suggested by the individual spectra in Figures 4 and 5 . These overplots show the magnitude of the variability in the 8600-8700 A region longward of the $\mathrm{CrH}$ bandhead and the possible variability in the $\mathrm{H}_{2} \mathrm{O}$ band longward of $9300 \AA$.

Variability has been reported previously in the spectra of other cool dwarfs. Tinney \& Tolley (1999) reported variability in the integrated light of two $30 \AA$ wide bandpasses centered at 8570 and $8725 \AA$ in the M9 brown dwarf LP 


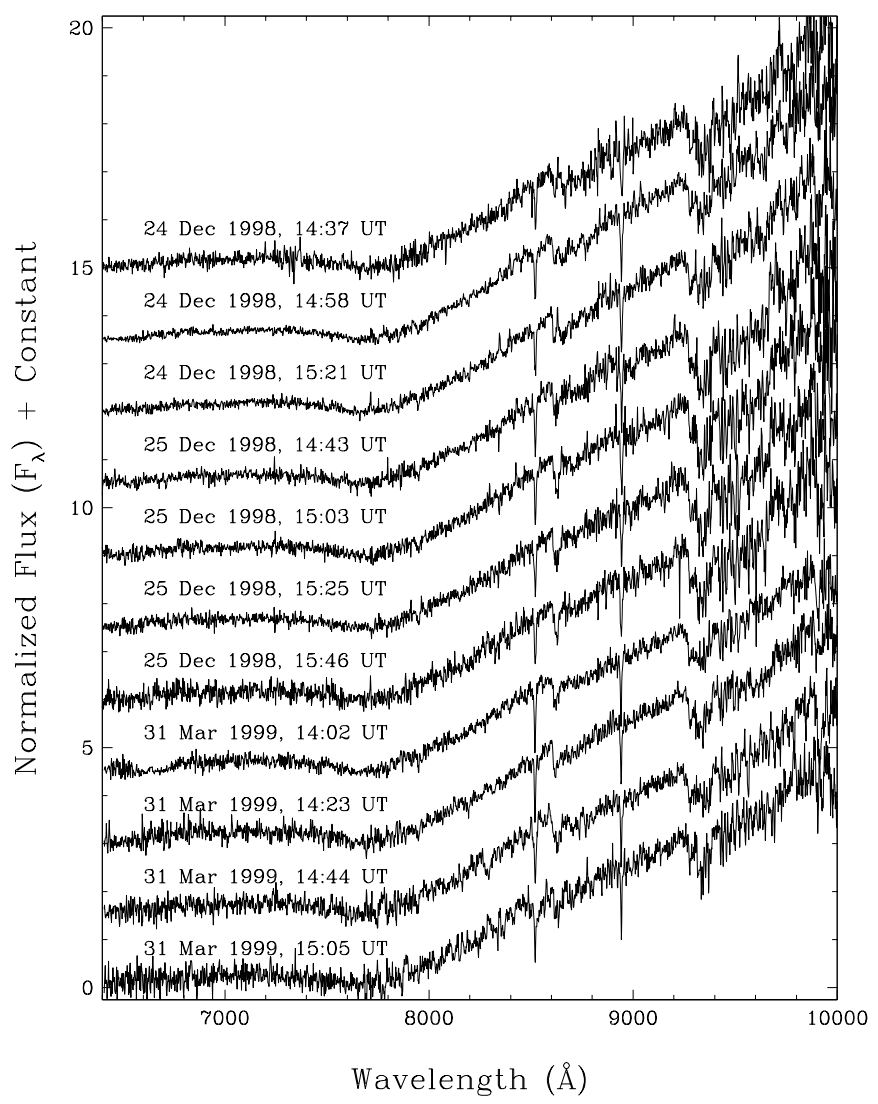

FIG. 3.-Spectra of $\mathrm{Gl} 584 \mathrm{C}$ from each of the 11 individual integrations taken on 1998 December 24, 1998 December 25, and 1999 March 31 (UT). Also listed are the start times of each integration. Each spectrum has been normalized to one at $8250 \AA$, and integral offsets have been used to separate the spectra vertically.

944-20. This is in the same spectral region where we see variability in G1 584C, and in the case of LP 944-20 the variation was detected in a $2 \mathrm{hr}$ time interval. Variability has also been observed by Nakajima et al. (2000) in the near-infrared spectrum of the T dwarf SDSSp J162414.37+002915.6. Here the time span was only 80 minutes, but slight changes were nonetheless seen in the depths of some features assumed to be $\mathrm{H}_{2} \mathrm{O}$. Bailer-Jones \& Mundt $(1999,2001)$ have also detected photometric variability at $I$ band in three L dwarfs and one late-M dwarf. In those studies, the L1.5 dwarf 2MASSW J1145572+231730 was shown to have a variability whose period does not repeat from one observing season to the next.

The variations seen in $\mathrm{Gl} 584 \mathrm{C}$ as well as the other cool dwarfs noted above may be a consequence of inhomogeneities in the photosphere. Such inhomogeneities would produce changes in the integrated light as the dwarf rotates. This hypothesis is supported, at least in part, by evidence that the rotation period of these cool dwarfs has a similar timescale as the observed variations, typically a few hours as measured via $v$ sin $i$ by Basri et al. (2000) and Schweitzer et al. (2001). What are these photospheric irregularities? An analogy to low-mass stars might suggest starspots - regions of cooler temperature and increased magnetic activity. An analogy to planets might suggest irregularly distributed "clouds" or bands in the atmosphere, the condensates blocking warmer layers below and producing chemical differentiation higher in the atmosphere. Bailer-Jones \& Mundt (2001) find that photometric variability is more common in L dwarfs than in late-M dwarfs; since chromospheric activity is seen to drop from late-M to early-L (Gizis et al. 2000), these authors suggest that starspots are unlikely to be the correct explanation. Moreover, the lack of significant periods in any of the confirmed variables leads them to conclude that if the inhomogeneities are due to surface features, they must evolve on timescales shorter than their observation baseline (typically 3-6 days). Additional observations are badly needed to quantify the variations - their amplitudes, their timescales, and the spectral features most affected-before we can begin to sort out the reasons behind them.

\section{AGE AND MASS ESTIMATES}

Because the primaries in both systems are bright, wellstudied $G$ dwarfs, we can use published observations to estimate their ages. ${ }^{5}$ We can then use those age estimates to constrain the masses of the $\mathrm{L}$ dwarfs themselves if we assume that the $\mathrm{G}$ dwarf primaries are coeval with their companions.

There are four main observational characteristics that can in principle be used to assign ages to solar-type stars: (1) chromospheric and coronal activity-in general the older the star, the less active it is ${ }^{6} ;(2)$ metallicity - this should be a direct reflection of the material, perhaps processed and recycled in previous generations of stars, from which it formed; (3) lithium abundance-for G dwarfs, the observed lithium abundance declines with time as the initial lithium is slowly destroyed and not replenished; (4) space velocity - on average this increases with age as objects in the galaxy undergo velocity boosts over their lifetimes due to encounters with giant molecular clouds.

All four of these measurements can also be intercompared on an ensemble of nearby stars to identify young, comoving, stellar kinematic groups. Establishing membership in a group is an even more valuable indicator of age since the properties of all members of the group can be used to determine a more reliable age for the group as a whole. In $\S 4.1$ we present the available data to establish an age for each system. In $\S 4.2$ we use those age determinations to estimate masses for the $\mathrm{L} d$ warf companions.

\subsection{Ages}

\subsubsection{Estimates from Chromospheric and Coronal Activity}

4.1.1.1. $X$-Ray Luminosities

There are several measurements that can be made to determine the level of chromospheric activity. One such indicator is the fraction of the star's luminosity emitted at $\mathrm{X}$-ray wavelengths. The logarithm of this quantity $\left(\log \left(L_{\mathrm{X}} / L_{\mathrm{bol}}\right)\right.$, Table 5) is plotted against $B-V$ color in Figure 7 for the G dwarfs Gl 417A, Gl 584AB, and the Sun. Also shown for comparison are the $\log \left(L_{\mathrm{X}} / L_{\mathrm{bol}}\right)$-values for members of the Pleiades and Hyades clusters as listed in

\footnotetext{
${ }^{5}$ For an overview of age dating for main-sequence stars of all types, see Lachaume et al. (1999).

${ }^{6}$ A sustained level of activity is expected in very close binary systems where tidal influences can counteract the trend of decreasing rotation with time. Such should not be the case for Gl 584AB since the pair is a relatively wide system of separation 16 AU (Mason et al. 1999). Here the observed measures of activity should still be a valid reflection of age.
} 

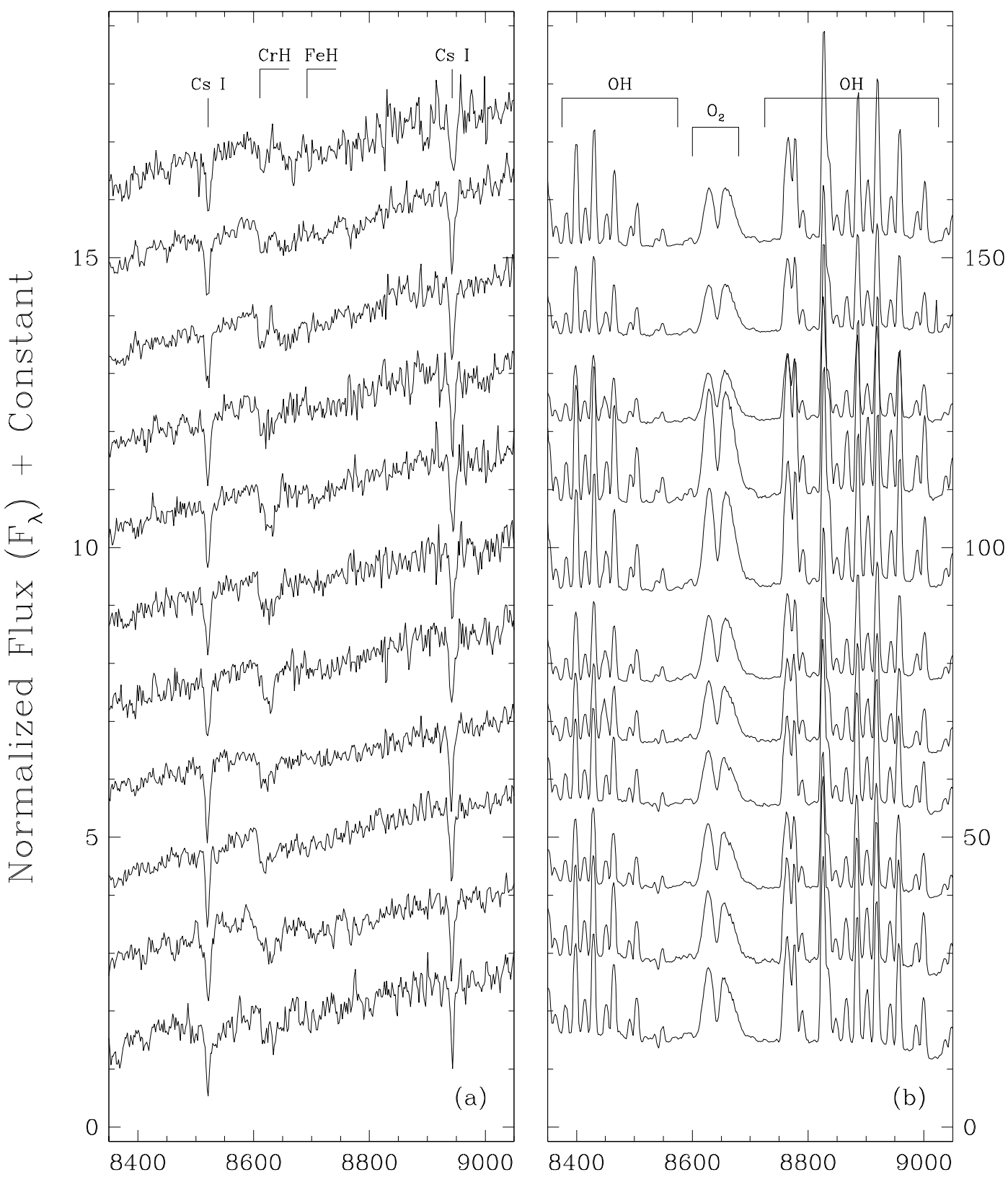

Wavelength $(\AA)$

FIG. 4. - (a) Detailed views of the 8350-9050 ̊ region for each of the 11 spectra shown in Fig. 3. Noteworthy spectral features are marked. (b) Night sky emission spectra for the same 11 integrations. These spectra are a forest of $\mathrm{OH}$ and $\mathrm{O}_{2}$ emission lines blended at this resolution. The scaling and ordering of the spectra are the same as in Fig. 3.

Stern, Schmitt, \& Kahabka (1995), Hempelmann et al. (1995), Micela et al. (1999), and Krishnamurthi et al. (1998). The figure shows that both G1 417A and G1 584AB are more X-ray active than the Sun with G1 417A more active than G1 584AB. Based on this plot alone, G1 584AB looks older than the average Hyad $(625 \pm 50 \mathrm{Myr}$; Perryman et al. 1998). Gl 417A appears somewhat younger than the Hyades but not inconsistent with the age of the Pleiades ( 125 Myr; Stauffer, Schultz, \& Kirkpatrick 1998).

Gaidos (1998) derives a relation between $\log \left(L_{\mathrm{X}} / L_{\mathrm{bol}}\right)$ and age for the evolving Sun using empirical relations for the time dependence of a sunlike star's luminosity, the time dependence of rotation rate, and the dependence of X-ray luminosity on rotation period:

$$
\begin{aligned}
\log \left(L_{\mathrm{X}} / L_{\mathrm{bol}}\right)= & -6.38-2.6 \alpha \log \left(\frac{t}{4.6}\right) \\
& +\log \left[1+0.4\left(1-\frac{t}{4.6}\right)\right],
\end{aligned}
$$

where $t$ is age in Gyr and $\alpha$ is the exponent in equation (3) below. Here equation (1) has been adjusted to yield the solar value listed in Table 5. Because both Gl 417A and Gl $584 \mathrm{AB}$ are very similar in spectral type to the Sun, we can use this relation to estimate crude ages for the two G dwarf 

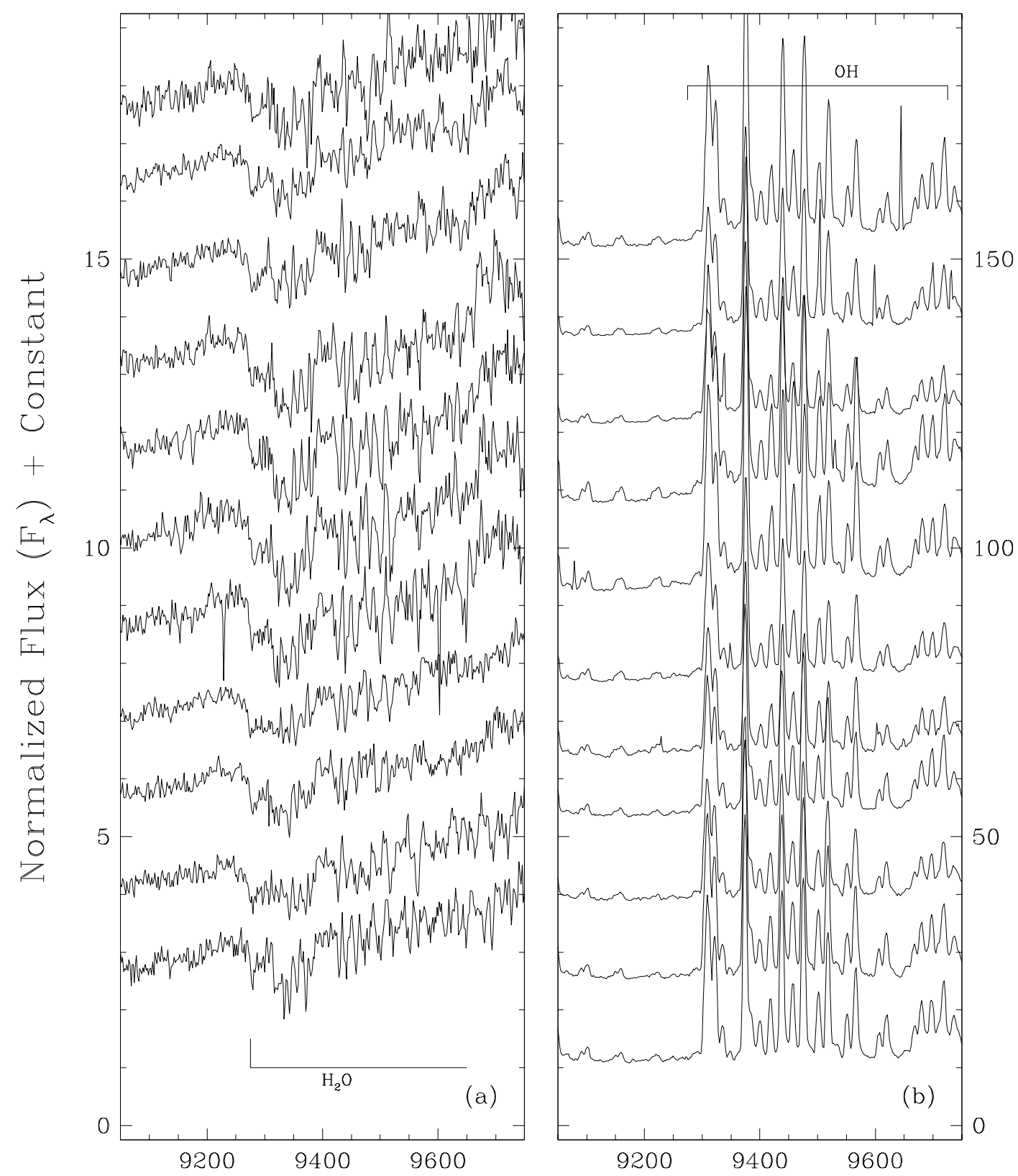

Wavelength $(\AA)$

FIG. 5.-Same as Fig. 4 except for the 9050-9750 Å region

systems: $80-250$ Myr for Gl 417A and $\sim 2$ Gyr for Gl $584 \mathrm{AB}$.

\subsubsection{Emission in $\mathrm{Ca}$ II}

A second activity indicator is the fraction of the total luminosity emerging as chromospheric emission in the $\mathrm{Ca}$ II $\mathrm{H}$ and $\mathrm{K}$ lines. The logarithm of this quantity $\left(\log R_{\mathrm{HK}}^{\prime}\right.$, Table 5) is plotted against $B-V$ color in Figure 8 for the $G$ dwarf systems listed in Table 5. Values for Hyades stars (Duncan et al. 1984) are also shown as well as mean values for several other clusters. G1 417A emits a larger percentage of its total flux in chromospheric emission than does G1 $584 \mathrm{AB}$. Whereas $\mathrm{Gl} 584 \mathrm{AB}$ has an $R_{\mathrm{HK}}^{\prime}$ index implying an age greater than the Hyades, Gl 417 is most likely of Hyades age or younger.
Donahue $(1993,1998)$ gives a relation between age and chromospheric emission. This relation is derived from observed $\log R_{\mathrm{HK}}^{\prime}$-values of stars in clusters spanning a wide range of ages and is valid for ages older than $10 \mathrm{Myr}$ :

$\log (t)=10.725-1.334 R_{5}+0.4085 R_{5}{ }^{2}-0.0522 R_{5}{ }^{3},(2)$ where $t$ is the age in Gyr and $R_{5}=\left(10^{5}\right) \log R_{\mathrm{HK}}^{\prime}$. Using this equation, we find an age estimate for G1 417A of $\sim 400 \mathrm{Myr}$ and for G1 584AB of $\sim 2.5 \mathrm{Gyr}$.

4.1.1.3. Rotational Period

Rotational period can also be used as an age indicator since rotation rate is coupled to the internal dynamo, which drives the level of chromospheric and coronal activity. The rotational period $\left(P_{\text {rot }}\right.$, Table 5$)$ is plotted against $B-V$ 


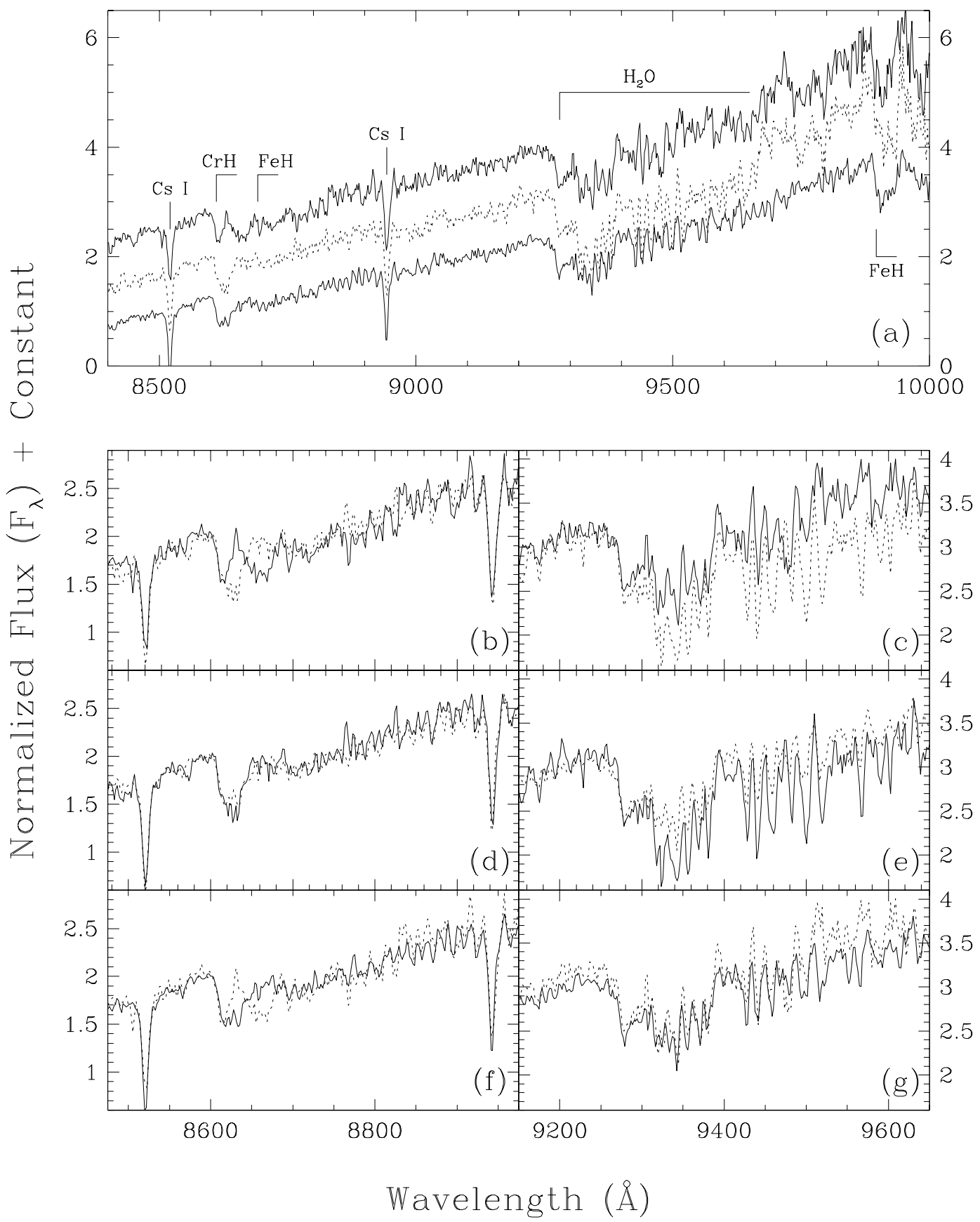

FIG. 6.-Comparisons of the nightly spectra of G1 584C. (a) The region between 8400 and $10000 \AA$ A. The middle spectrum (dashed line) is the summed spectrum taken on 1998 December 25 and normalized to one at $8250 \AA \AA$. Above and below this are the summed spectra for 1998 December 24 (solid line, offset 0.75 upward) and 1999 March 31 (solid line, offset 0.75 downward). The strongest spectral features are marked. ( $b, d, f$ ) Pairwise overplots of the spectra between 8475 and $8975 \AA .(c, e, g)$ Pairwise overplots of the spectra between 9150 and $9650 \AA$. The pairs shown in each panel are $(b-c) 1998$ December 24 (solid line) and 1998 December 25 (dashed line); (d-e) 1998 December 25 (solid line) and 1999 March 31 (dashed line); $(f-g) 1999$ March 31 (solid line) and 1998 December 24 (dashed line).

color in Figure 9. The locus occupied by Hyades and Pleiades stars is also shown (Stern et al. 1995; Hempelmann et al. 1995; Krishnamurthi et al. 1998). The rotation period of Gl 417A implies that it is at least as young as the Hyades, while that of Gl 584AB implies an age older than the Hyades.

The period of rotation is thought to increase as a power law over time:

$$
P_{\text {rot }} \propto t^{\alpha},
$$

where the exponent most likely falls in the range $1 / 2 \lesssim \alpha \lesssim$ 1/e (Skumanich 1972; Walter \& Barry 1991). If we take the 25.3 day rotation period of the Sun along with a solar age of 4.6 Gyr, we can use equation (3) to estimate ages for the $\mathrm{G}$ dwarf systems of 150-400 Myr for Gl 417A and $850 \mathrm{Myr}-$ $1.35 \mathrm{Gyr}$ for Gl 584AB.

\subsubsection{Chromospheric Variations}

Finally, the variation in chromospheric activity can also be used as a crude chronometer of age for solar-type stars. 
TABLE 5

Compiled Data on the G Dwarf Systems with Comparison to the Sun

\begin{tabular}{|c|c|c|c|c|c|c|}
\hline \multirow[b]{2}{*}{ PROPERTY } & \multicolumn{2}{|c|}{ Gl 417A } & \multicolumn{2}{|c|}{ Gl 584AB } & \multicolumn{2}{|c|}{ Sun } \\
\hline & Data & Reference & Data & Reference & Data & Reference \\
\hline Spectral type ....... & G0 V & 1 & $\mathrm{G} 1 \mathrm{~V}+\mathrm{G} 3 \mathrm{~V}^{\mathrm{a}}$ & 6 & G2 V & - \\
\hline$B-V \ldots \ldots \ldots \ldots \ldots$ & 0.60 & 2 & 0.58 & 7 & 0.66 & 11 \\
\hline \multicolumn{7}{|l|}{ Activity: } \\
\hline $\log \left(L_{\mathrm{X}} / L_{\mathrm{bol}}\right) \ldots \ldots$ & -4.55 & 2 & -5.89 & 8 & -6.38 & 11 \\
\hline $\log \left(R_{\mathrm{HK}}^{\prime}\right) \ldots \ldots \ldots$ & -4.40 & 2 & -4.77 & 9 & $-4.93^{\mathrm{c}}$ & 12 \\
\hline$P_{\text {rot }}$ (days) ........ & 7.6 & 2 & 13.6 & 10 & 25.3 & 11 \\
\hline Period ............ & Variable & 3 & Variable & 3 & Cyclical & 3 \\
\hline \multicolumn{7}{|l|}{ Metallicity: } \\
\hline$[\mathrm{Fe} / \mathrm{H}] \ldots$ & -0.01 & 4 & -0.20 & 4 & 0.00 & - \\
\hline \multicolumn{7}{|l|}{ Lithium abundance: } \\
\hline $\log N_{\mathrm{Li}}^{\mathrm{b}} \ldots \ldots \ldots \ldots$ & 2.38 & 5 & $2.61 \pm 0.10$ & 7 & $0.95 \pm 0.10$ & 13 \\
\hline \multicolumn{7}{|l|}{ Kinematics: } \\
\hline$U\left(\mathrm{~km} \mathrm{~s}^{-1}\right)^{\mathrm{d}} \ldots \ldots$ & $-6.7 \pm 0.3$ & 14 & $+14.6 \pm 1.5$ & 9 & $\ldots$ & - \\
\hline$V\left(\mathrm{~km} \mathrm{~s}^{-1}\right) \ldots \ldots \ldots$ & $-11.7 \pm 0.5$ & 14 & $-4.6 \pm 0.4$ & 9 & $\ldots$ & - \\
\hline$W\left(\mathrm{~km} \mathrm{~s}^{-1}\right) \ldots \ldots$ & $-4.1 \pm 0.2$ & 14 & $-12.5 \pm 0.6$ & 9 & $\ldots$ & - \\
\hline
\end{tabular}

${ }^{\text {a }}$ The composite spectral type of this close binary $\left(\rho=1^{\prime \prime} .034\right.$ at $\left.\theta=29^{\circ} .9\right)$ is given as G2 V by Struve \& Franklin 1955. Edwards 1976 estimates the individual types are G1 V and G3 V based on the combined spectral type and known magnitude difference between components.

${ }^{\mathrm{b}}$ The primordial lithium abundance of the sun, based on analysis of carbonaceous chondrites, is $\log N_{\mathrm{Li}}=3.34$ \pm 0.07 (Reeves \& Meyer 1978).

c This is an average of the values at Maunder minimum $(-5.10)$ and solar maximum $(-4.75)$.

${ }^{\mathrm{d}}$ Here $U$ is positive in the direction of the Galactic center.

REFERENCES.- (1) Bidelman 1951; (2) Gaidos 1998; (3) Baliunas et al. 1995; (4) Soderblom 1983; (5) Duncan 1981; (6) Edwards 1976; (7) Soderblom et al. 1993b; (8) Schmitt et al. 1990; (9) Soderblom \& Mayor 1993; (10) Hale 1994; (11) Maggio et al. 1987; (12) Henry et al. 1996; (13) Müller et al. 1975; (14) Gaidos et al. 2000.

Stars with low-amplitude variability generally have cyclical periods and are old stars like the Sun. G dwarfs of younger age ( $\sim 1-2$ Gyr) show moderate-amplitude variability and occasional cyclical periods. Much younger stars show larger variability with no well-defined periodicity. As listed in Table 5, both G1 417A and G1 584AB show variable periodicities and this implies, as do the other activity indicators discussed above, that these $G$ dwarfs are considerably younger than the Sun.

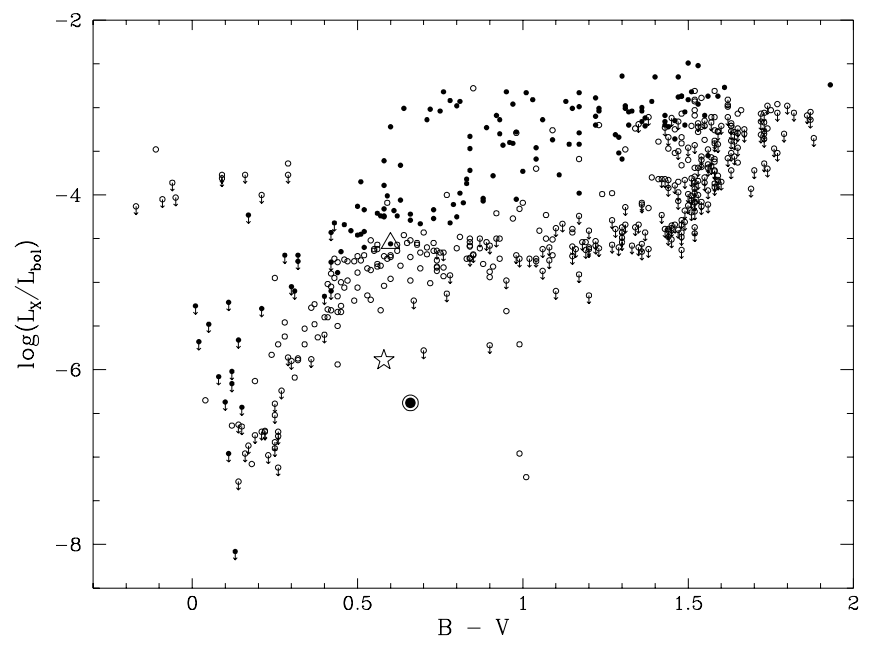

FIG. 7. $-\log \left(L_{\mathrm{X}} / L_{\text {bol }}\right)$ vs. $B-V$. Data for the Pleiades (age $\left.\sim 125 \mathrm{Myr}\right)$ are shown by the small solid circles. Data for the Hyades (age $\sim 625 \mathrm{Myr}$ ) are shown by the small open circles. Upper limits are denoted by downward arrows. The position of the G dwarf Gl 417A is shown by the large open triangle, and that of the $\mathrm{G}$ dwarf double $\mathrm{Gl} 584 \mathrm{AB}$ is shown by the large open star. The location of the Sun (age $\sim 4.6 \mathrm{Gyr}$ ) is shown by the large encircled dot. The sources for these data are given in the text.

\subsubsection{Estimates from Metallicity}

The values of $[\mathrm{Fe} / \mathrm{H}]$ in Table 5 show that Gl 417A has solar metallicity while $\mathrm{Gl} 584 \mathrm{AB}$ is more metal poor by 0.2 dex. Figure 10 shows mean $[\mathrm{Fe} / \mathrm{H}]$-values for clusters and moving groups of known age, as measured from $F$ and $G$ stars by Boesgaard (1989), Boesgaard \& Friel (1990), and Friel \& Boesgaard (1992). This figure demonstrates that there is little correlation between metallicity and age, leading Boesgaard (1989) to the conclusion that the principal factor determining a cluster's metal content is not its

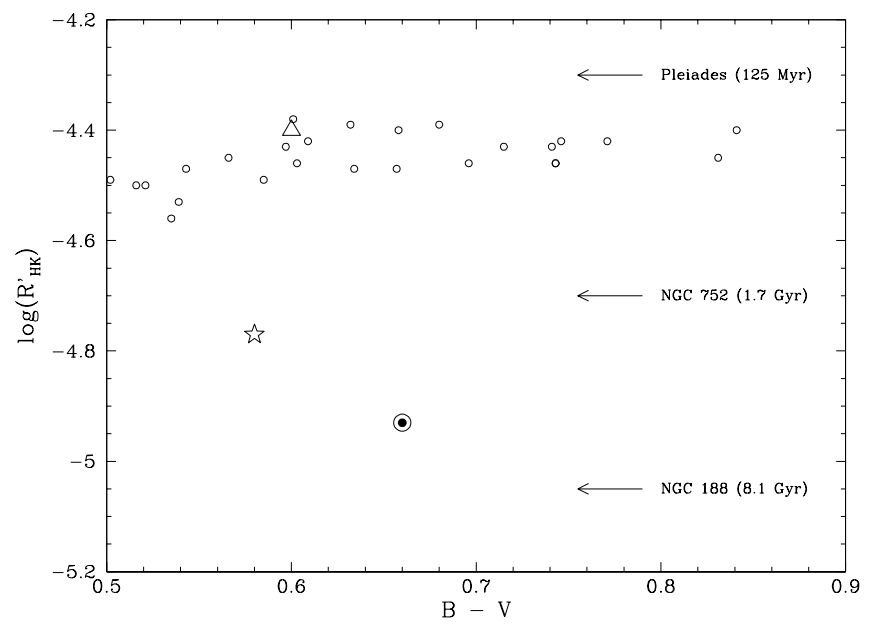

FIG. 8. $-\log \left(R_{\mathrm{HK}}^{\prime}\right)$ vs. $B-V$. Data for the Hyades (age $\left.625 \mathrm{Myr}\right)$ are shown by the small open circles and are taken from Duncan et al. (1984). Also shown are mean $\log \left(R_{\mathrm{HK}}^{\prime}\right)$-values and ages for the Pleiades, NGC 752, and NGC 188 as taken from Fig. 1 of Donahue (1998). The locations of Gl $417 \mathrm{~A}$, G1 584AB, and the Sun are shown using the same symbols as those in Fig. 7. 


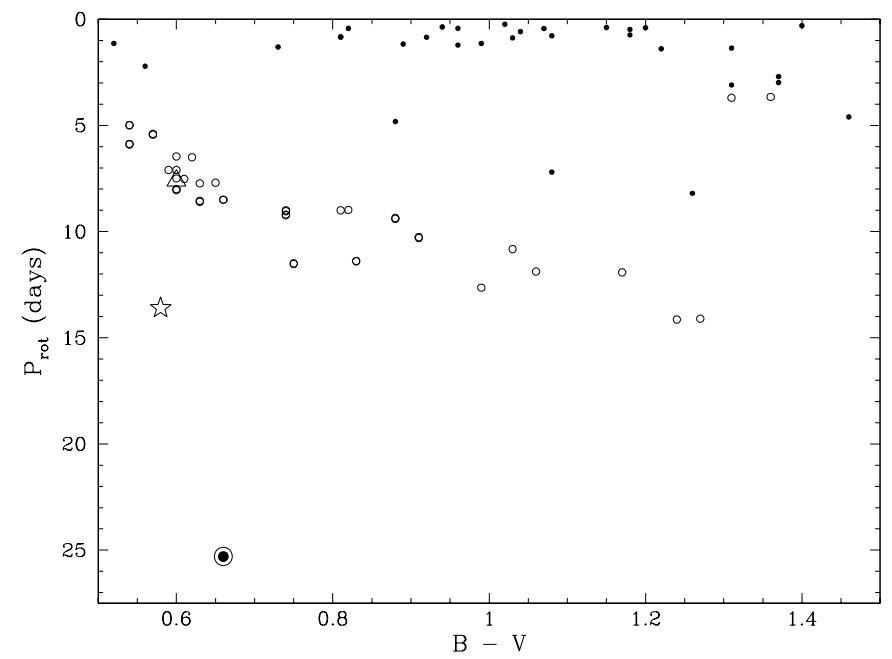

FIG. 9. $-P_{\text {rot }}$ vs. $B-V$. Symbols are the same as in Fig. 7. The sources for these data are listed in the text.

age but its position in the Galaxy. Edvardsson et al. (1993, see their Fig. 31) show that the age-metallicity relation for field stars also shows very large scatter. Therefore, the metallicities of $\mathrm{G}$ dwarfs cannot be used as chronometers but may be suggestive of membership in nearby clusters or moving groups.

\subsubsection{Estimates from Lithium Abundance}

In the study of stellar atmospheres, lithium is an important element because (1) it is easily fused in stellar interiors and (2) it does not occur as a stable by-product of any normal thermonuclear reaction (Herbig 1965; Bodenheimer 1965). Hence, a star's current lithium content is just the fraction of its nascent lithium that it has not yet destroyed. Thus, by assuming an initial lithium abundance and by measuring the current abundance, the age of the star can be estimated.

However, the details of how a star recycles the material near its photosphere (i.e., those layers visible to spectrographs) into deeper layers where lithium destruction

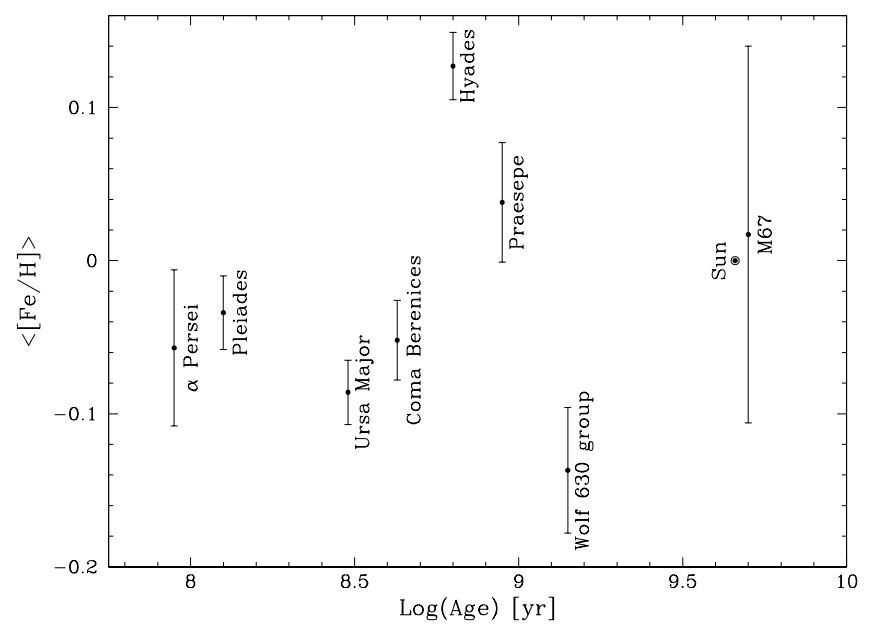

FIG. 10.-Mean $[\mathrm{Fe} / \mathrm{H}]$ vs. $\log$ age for various clusters and moving groups. Data are from Friel \& Boesgaard (1992). More recent age determinations for $\alpha$ Persei (90 Myr, Stauffer et al. 1999), the Pleiades (125 Myr, Stauffer et al. 1998), the Hyades (625 Myr, Perryman et al. 1998), and Praesepe (900 Myr, Hambly et al. 1995) have been employed here. occurs are complex and vary as a function of mass. $\mathrm{K}$ and early-M dwarfs, for example, are believed to be fully convective and as a result of convective turnover will destroy all of their primordial lithium. For brown dwarfs below $0.06 M_{\odot}$, the interior never reaches temperatures sufficient for lithium destruction, so these objects retain their full complement of primordial lithium on timescales of less than $100 \mathrm{Myr}$ (Magazzù et al. 1993). In a mid-to-late $\mathrm{F}$ dwarf the convection zone is too shallow to allow nuclear reactions to destroy lithium at its base. However, microscopic diffusion of $\mathrm{Li}$ atoms below the convection zone will, slowly with time, deplete the star's lithium reserve. For these stars, lithium abundance is a good tracer of age (Boesgaard 1991).

The lithium abundance, $\log (N(\mathrm{Li}))$, has been measured for mid-to-late $\mathrm{F}$ dwarfs in several well studied clusters. These mean values are plotted as a function of cluster age in Figure 11. Boesgaard (1991) represents the relationship by

$$
\log [N(\mathrm{Li})]=-0.30 \log (t)+5.33,
$$

where $\log [N(\mathrm{Li})]$ is on the scale where $\log [N(\mathrm{H})]=12.00$ and $t$ is the age of the $F$ star in Gyr. In early-type G dwarfs, like Gl 417A and G1 584AB, the rate of lithium diffusion is higher than for mid-to-late $F$ stars, so the values of $\log [N(\mathrm{Li})]$ listed in Table 5 need to be adjusted for use in equation (4). In Figure 1 of Boesgaard (1991), it is shown that the $\log [N(\mathrm{Li})]$-values for stars of Hyades age decline by $\sim 0.3$ dex from mid-F to G0. Between mid-F and G1, the difference is $\sim 0.6$ dex. Figure $9 b$ of Soderblom et al. (1993a) shows that for stars of Pleiades age, $\log [N(\mathrm{Li})]$ is roughly constant from mid-F to G1. As further reference, the dependence of lithium abundance on age and on spectral type $\left(T_{\text {eff }}\right)$ is especially well presented in Figure 4 of Gaidos, Henry, \& Henry (2000).

We can therefore assume corrections to the lithium abundances for the G0 dwarf G1 417A and the $\sim \mathrm{G} 1$ dwarf pair Gl $584 \mathrm{AB}$, plug those values into equation (4), and determine for which values the resulting age is consistent with the size of the correction assumed. When we do this, we find that equation (4) predicts self-consistent ages of $\sim 600 \mathrm{Myr}$ for Gl 417A and $\sim 300 \mathrm{Myr}$ for G1 584AB. For late-F stars in a single cluster, however, star-to-star lithium abundances can vary by \pm 0.4 dex in $\log [N(\mathrm{Li})]$. Moreover, as Figure

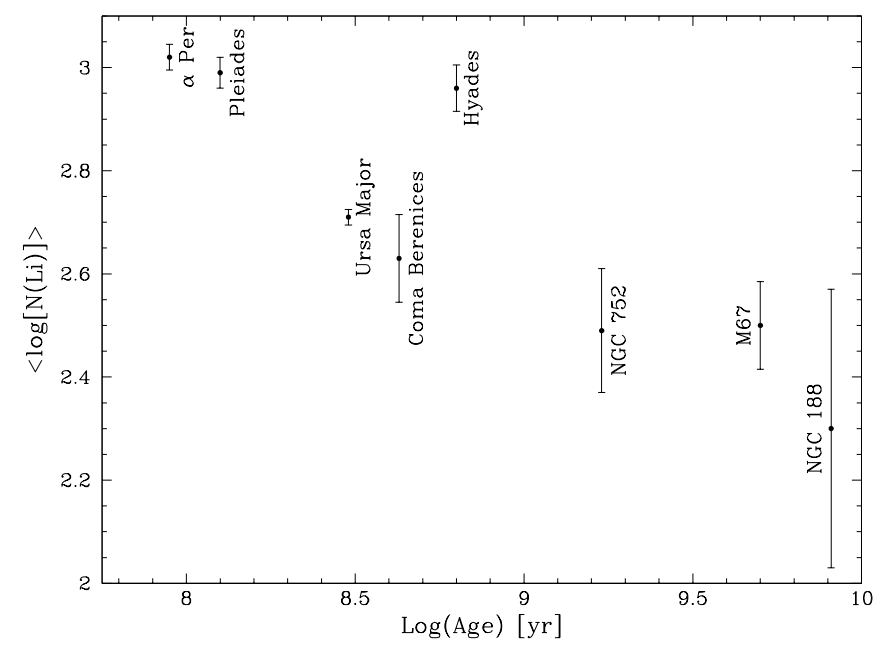

FIG. 11.- Mean $\log [N(\mathrm{Li})]$-values for mid-to-late $\mathrm{F}$ dwarfs plotted against age for various clusters and moving groups. Data are taken from Boesgaard (1991) but supplemented with more recent age determinations as detailed in the caption to Fig. 10. 
TABLE 6

Age Estimates for Each System

\begin{tabular}{|c|c|c|}
\hline Property & $\begin{array}{c}\text { Gl } 417 \mathrm{~A} \\
\text { (1) }\end{array}$ & $\begin{array}{c}\text { Gl } 584 \mathrm{AB} \\
\text { (2) }\end{array}$ \\
\hline X-ray luminosity. & $80-250 \mathrm{Myr}$ & $\sim 2 \mathrm{Gyr}$ \\
\hline $\mathrm{Ca}$ II emission ..... & $\sim 400 \mathrm{Myr}$ & $\sim 2.5 \mathrm{Gyr}$ \\
\hline Rotational period $\ldots \ldots \ldots \ldots \ldots \ldots$ & $150-400 \mathrm{Myr}$ & $850 \mathrm{Myr}-1.35 \mathrm{Gyr}$ \\
\hline Chromospheric variations ........ & Young & Young \\
\hline Metallicity ..................... & (See text) & (See text) \\
\hline Lithium abundance ............... & $600::$ Myr & $300::$ Myr \\
\hline Kinematics $\ldots \ldots \ldots \ldots \ldots \ldots \ldots$ & Young disk & Young disk \\
\hline Moving group membership....... & Local Association? & None \\
\hline Final age estimate .... & $80-300 \mathrm{Myr}$ & $1-2.5 \mathrm{Gyr}$ \\
\hline
\end{tabular}

11 shows, the metal-rich Hyades falls significantly above the mean relation suggested by the other clusters, so the rate of lithium depletion may also have a dependence on metallicity. Another complication is that lithium depletion may also depend on the rotational history of the star (Ventura et al. 1998). Thus, these lithium-based age estimates for G1 $417 \mathrm{~A}$ and G1 584AB should be considered as very crude estimates only.

\subsubsection{Estimates from Kinematics}

The $U, V, W$ space motions of Gl 417A and G1 584AB place them squarely in the "young disk" as defined by Eggen (1963) and Leggett (1992). (Note that those papers use the left-handed coordinate system-i.e., where $U$ is defined as positive away from the Galactic center-as opposed to the right-handed coordinate system employed here.) Space motion is a poor indicator of age for individual stars. However, while old stars can have low space motions relative to the Local Standard of Rest (LSR), very few young stars have high velocities. Thus, the observed motions (Table 5) are consistent with, and even suggestive of, relatively young ages for both stellar systems considered here.

\subsubsection{Estimates from Inclusion in Moving Groups}

The individual age estimates from above are listed in Table 6 for both of the $\mathrm{G}$ dwarf primaries. Using this infor-

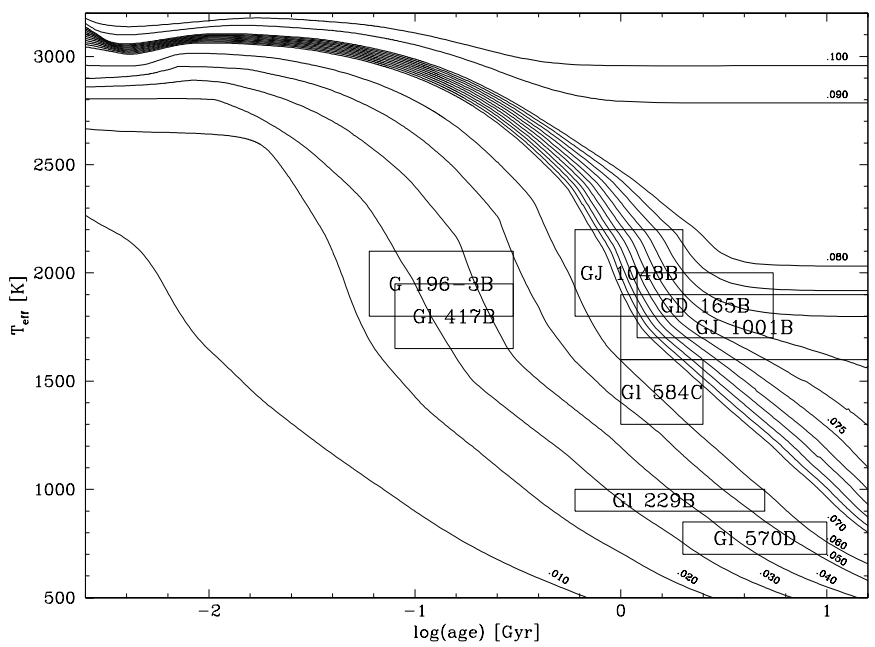

Fig. 12.-Theoretical plots for low-mass stars and brown dwarfs from Burrows et al. (1997). The mass, in $M_{\odot}$, is shown along each track. Positions are shown for each of the $\mathrm{L}$ and $\mathrm{T}$ dwarf companions listed in Tables $7 \mathrm{~A}$ and $7 \mathrm{~B}$. mation, we can determine whether either is a likely member of a known moving group.

Gl 417A has been listed by Gaidos (1998) as a possible member of the Local Association, which appears to comprise a kinematically coherent stream of clusters and associations including $\alpha$ Persei and the Pleiades. These stars have ages substantially less than $300 \mathrm{Myr}$, most having ages of 20-150 Myr (Jeffries 1995). The space motion of Gl 417A (Table 5) is in reasonable agreement with the space motion ${ }^{7}$ of stars thought to belong to the Group, $U, V, W=-10$, $-21,-13$ (Jeffries 1995), but the three-dimensional dispersion is a very large $9.9 \mathrm{~km} \mathrm{~s}^{-1}$. The metallicity is also in good agreement with $\alpha$ Persei and the Pleiades (Fig. 10). It is clear, though, that an age as young as $20 \mathrm{Myr}$ for this star is not supported by the evidence summarized in Table 6 . If this star is a member of the Local Association, it would be one of the older members. Based on the evidence in Table 6 and the suggestion of Local Association membership, we assign an age of 80-300 Myr to Gl 417A.

Gl 584AB has been listed previously as a possible member of the Ursa Major Group, which has a traditional age of $300 \mathrm{Myr}$. Although G1 584AB is located on the sky near stars comprising the core of the UMa Group and has a similar distance-18.6 pc for Gl 584AB and $21.7 \mathrm{pc}$ for the Group itself (Soderblom \& Mayor 1993) - the age estimates given above and as summarized in Table 6 indicate that this $\mathrm{G}$ dwarf pair is significantly older than $300 \mathrm{Myr}$. Soderblom $\&$ Mayor (1993) arrive at the same conclusion based on chromospheric activity comparisons alone. We also note that the metallicity of $\mathrm{Gl} 584 \mathrm{AB},[\mathrm{Fe} / \mathrm{H}]=-0.20$, is slightly lower than the mean of the Group, $[\mathrm{Fe} / \mathrm{H}]=$ $-0.086 \pm 0.021$, and significantly lower than the $[\mathrm{Fe} / \mathrm{H}]$ values for all eight UMa Group members with robust measures in Boesgaard \& Friel (1990). A comparison of the space motion of G1 584AB with the mean motion of stars forming the group nucleus, $U, V, W=+12.6,+2.1,-8.0$ (Soderblom \& Mayor 1993), also indicates that Gl 584AB is probably not a member since this star falls outside of the measured three-dimensional dispersion of $1.7 \mathrm{~km} \mathrm{~s}^{-1}$. We use the individual age estimates in Table 6 to derive an age range of 1-2.5 Gyr for this system, substantially older than the age of the Ursa Major Group.

${ }^{7}$ All $U, V, W$ space velocities reported here are corrected to the LSR using a solar motion of $U, V, W=+10.4,+14.8,+7.3 \mathrm{~km} \mathrm{~s}^{-1}$, as described in Johnson \& Soderblom (1987). 


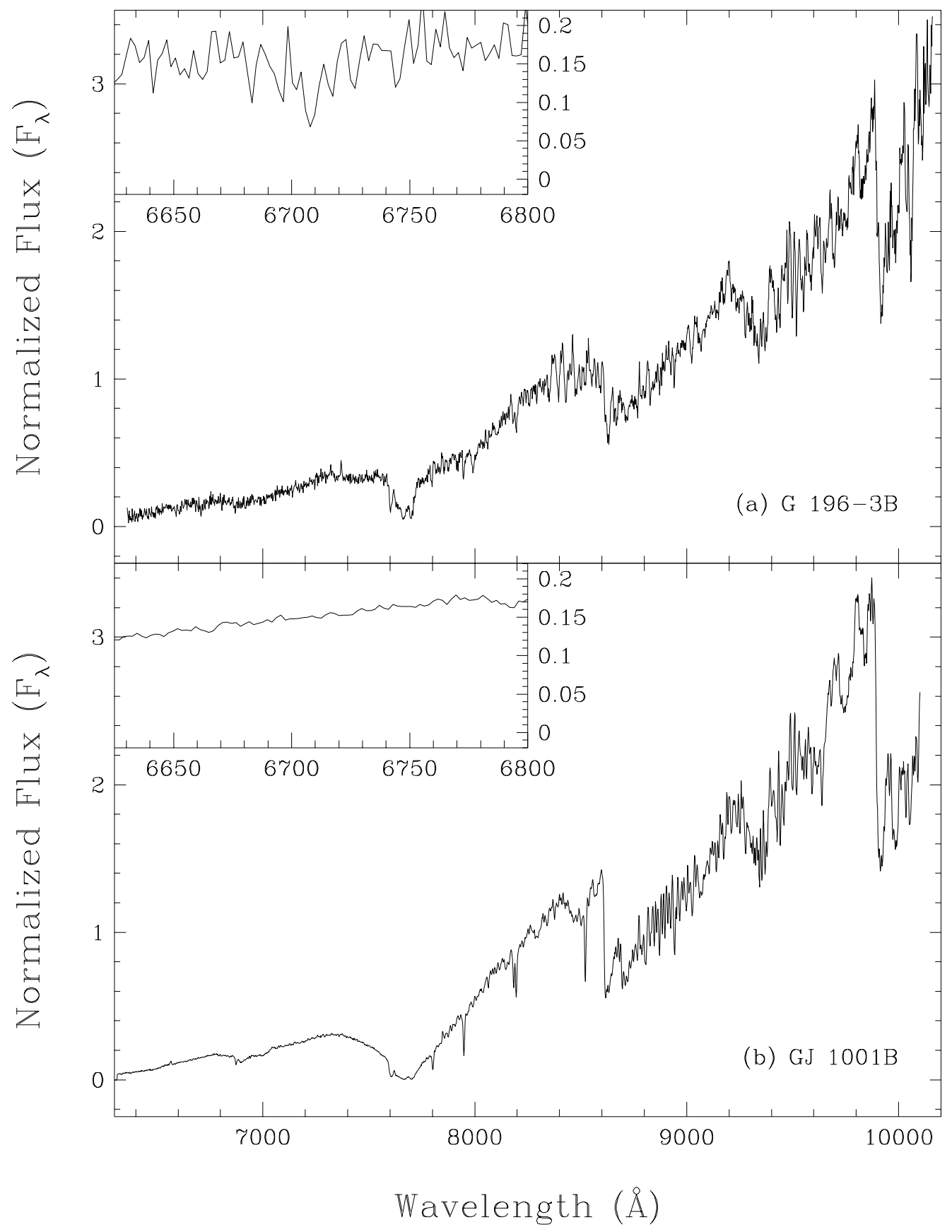

Fig. 13. - Spectra of two other L dwarf companions. (a) A $6000 \mathrm{~s}$ sum of the G 196-3B observations listed in Table 3. Shown in the inset is a detailed view of the spectrum between 6600 and $6800 \AA$ to highlight the lithium absorption at $6708 \AA$. (b) The $1200 \mathrm{~s}$ spectrum of GJ 1001B. The inset shows a detailed view near $6708 \AA$ A demonstrating the lack of lithium absorption. In both the full panels and the insets, spectra have been normalized to one at $8250 \AA$.

\subsection{Masses}

The spectral type of each L dwarf companion can be used to get a crude estimate of temperature. Using these temperature estimates and the age estimates from above, both companions can be placed on the theoretical H-R diagram and masses estimated. For Gl 417B its spectral type of L4.5 $\mathrm{V}$ would place its temperature between 1600 and $1800 \mathrm{~K}$ based on the best current estimates (see, e.g., Kirkpatrick et al. 2000 and Basri et al. 2000). For G1 584C its spectral type of L8 V would place its temperature between 1300 and 1600 $\mathrm{K}$, though a temperature nearer $1300 \mathrm{~K}$ is favored based on simple physical arguments (Kirkpatrick et al. 2000; Reid et al. 2001).
Using the nongray atmospheres computed by Burrows et al. (1997), we can estimate masses for the two L dwarf companions. As shown in Figure 12, this gives $M=0.035$ $\pm 0.015 M_{\odot}$ for Gl 417B and $M=0.060 \pm 0.015 M_{\odot}$ for Gl 584C. Note that the less massive object has an earlier spectral type (is hotter) due to its much younger age.

\section{COMPARISON TO OTHER L AND T DWARF COMPANIONS}

Several other $\mathrm{L}$ and $\mathrm{T}$ dwarf companions are also now known and can be compared to G1 417B and G1 584C. For three of these-GJ 1048B, G 196-3B, and GJ 1001B (LHS 102B) - we have obtained far red spectra to place them on 


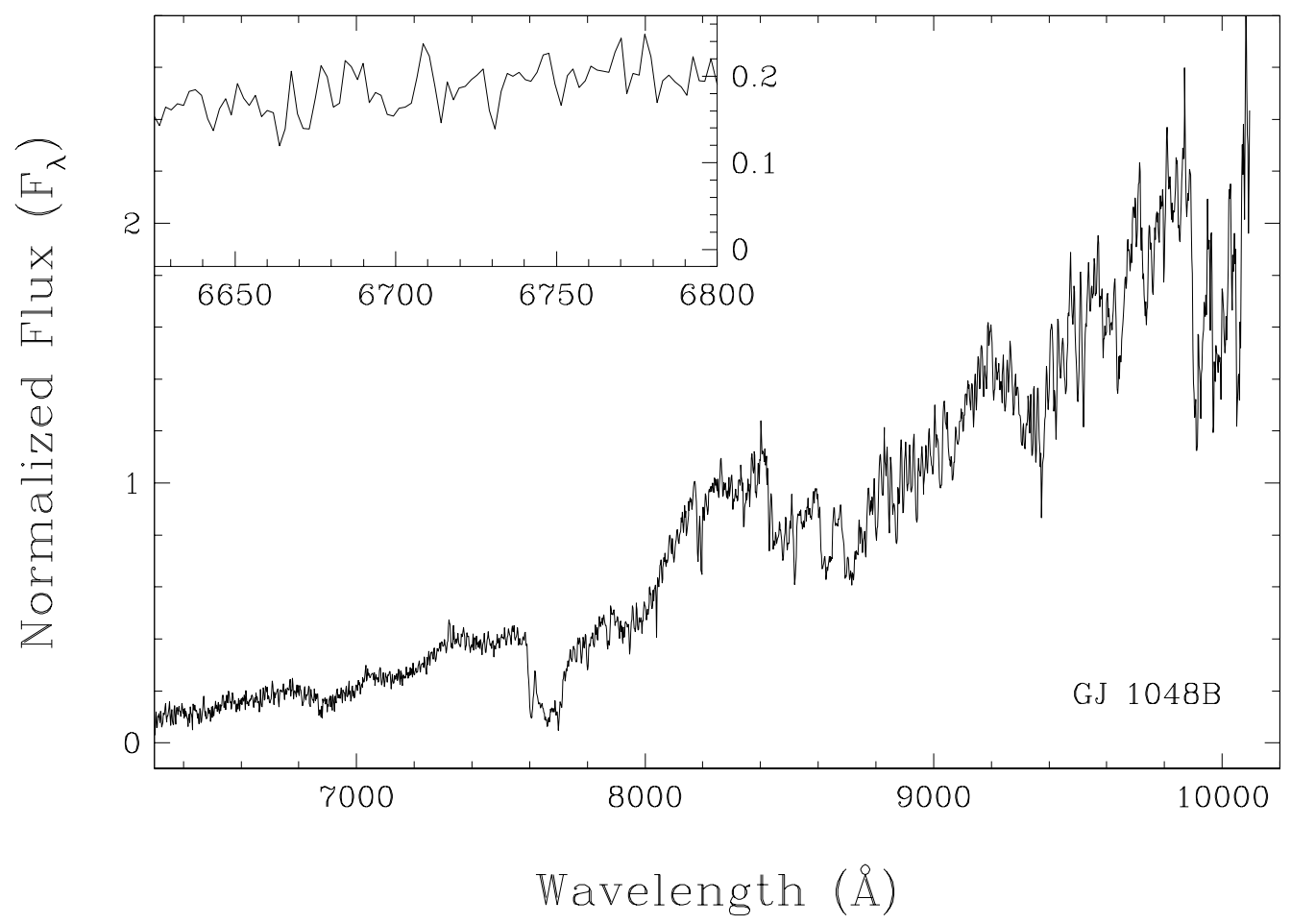

FIG. 14.- Spectrum of another L dwarf companion, GJ 1048B, as listed in Table 3. As with Figs. 2 and 13, the inset shows the area between 6600 and 6800 $\AA$. In the full panel and the inset, the spectrum has been normalized to one at $8250 \AA$.

the same classification system as the other L dwarf companions. Spectra of these three objects were obtained with LRIS at Keck using the setup described in $\S 3.2$. An observing journal for these three observations is given in the lower portion of Table 3, and reduced spectra are shown in Figures 13 and 14. Using the classification scheme of Kirkpatrick et al. (1999b), we assign types of L1 V to GJ 1048B, L2 V to G 196-3B, and L5 V to GJ 1001B. (Spectra of the latter two objects are also shown in Martín et al. 1999.) We note that GJ 1048B shows no $\mathrm{Li}$ I $6708 \AA$ absorption $(\mathrm{EW}<1 \AA$ ) or $\mathrm{H} \alpha$ emission $(\mathrm{EW}<1 \AA$ ) $)$ G 196-3B shows Li I absorption with $\mathrm{EW} \approx 6 \AA$ but no measurable $\mathrm{H} \alpha$ emission $(\mathrm{EW}<0.5 \AA$ ). GJ 1001B shows no Li I absorption $(\mathrm{EW}<0.2 \AA)$ but shows weak $\mathrm{H} \alpha$ emission $^{8}$ with $\mathrm{EW} \approx 1.5 \AA$.

Table 7A lists the physical data ${ }^{9}$ for each of the $\mathrm{L}$ dwarf companions, and Table 7B lists data for the two known $\mathrm{T}$ dwarf companions. Using our model-independent ages, we can plot these published companions on the theoretical $\mathrm{H}-\mathrm{R}$ diagram of Figure 12. As with Gl 417B and G1 584C, temperatures for the $\mathrm{L}$ dwarfs of Table $7 \mathrm{~A}$ have been estimated from spectral types, with the temperature scales of Basri et al. (2000) and Kirkpatrick et al. (2000) used to bracket the most likely ranges. Temperature estimates for the $\mathrm{T}$ dwarf companions are taken from the literature.

\footnotetext{
${ }^{8}$ This object is the latest type $\mathrm{L}$ dwarf for which $\mathrm{H} \alpha$ emission has been observed (see Kirkpatrick et al. 2000), thanks in part to the excellent signal-to-noise in this spectrum.

${ }^{9}$ We have used only the age estimates for the primary stars to assign ages for each companion. As a result, our age estimates differ from some published values that incorporate comparisons between the properties of the companions and brown dwarf theoretical models. Our approach allows us to use age diagnostics deduced from the companion as independent tests of ages derived from the primary alone.
}

Figure 12 shows that these $\mathrm{L}$ and $\mathrm{T}$ dwarf companions span a mass range from $\sim 0.035$ to $\sim 0.075 M_{\odot}$. The final mass estimates are given in Tables $7 \mathrm{~A}$ and $7 \mathrm{~B}$. It is interesting to note that the dwarfs G 196-3B, Gl 417B, and Gl 229B have nearly equal masses and thus to first order can be thought of as snapshots of a $0.035 M_{\odot}$ brown dwarf at three different stages during its cooling history. For an age even younger than that of the Pleiades, such an object would be a late $M$ dwarf.

For objects as cool as L dwarfs, the presence of lithium indicates a mass below $\sim 0.060 M_{\odot}$. Although we have not yet used the presence or absence of lithium to constrain the ages or masses for the companions above, we see that the results are self-consistent. That is, for $\mathrm{L}$ dwarf companions without lithium, all have independent mass estimates placing them above $\sim 0.060 M_{\odot}$. Except for G1 584C, all of the $\mathrm{L}$ dwarfs with lithium have mass estimates placing them below $\sim 0.060 M_{\odot}$. The lithium detection in G1 584C favors an age in the younger part of our estimated age range and a mass in the lower half of our mass range.

\section{CONCLUSIONS}

We have presented astrometric evidence that the $G$ dwarfs G1 417A and Gl 584AB both have very low luminosity, common proper motion companions. Spectroscopy shows that both are L dwarfs and both have strong lithium absorption lines, confirming that they are brown dwarfs. The cooler object, G1 584C, is a very late L dwarf exhibiting band strength variations in its spectrum, perhaps a sign of changing "weather" in the brown dwarf atmosphere. Because the primaries of both systems are previously well studied, many parameters such as absolute magnitude, age, and metallicity can already be assigned to the L dwarfs themselves. The L4.5 dwarf Gl 417B has $M_{J}=12.9$, is between 0.08 and $0.3 \mathrm{Gyr}$ old, and has $[\mathrm{Fe} / \mathrm{H}]=-0.01$. 


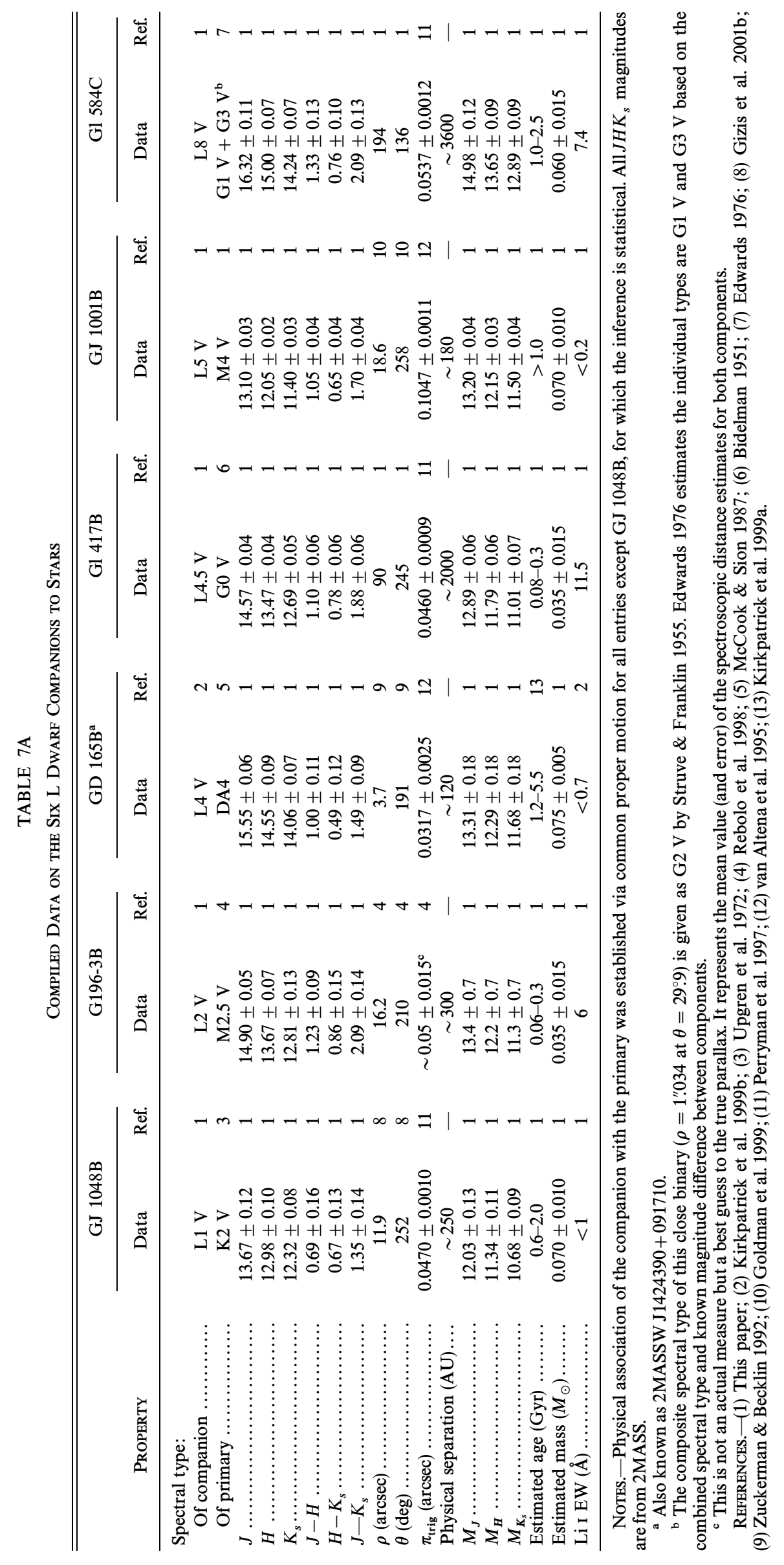


TABLE 7B

Compiled Data on the Two T Dwarf Companions to Stars

\begin{tabular}{|c|c|c|c|c|}
\hline \multirow[b]{2}{*}{ PROPERTY } & \multicolumn{2}{|c|}{ G1 229B } & \multicolumn{2}{|l|}{ G1 570D } \\
\hline & Data & Ref. & Data & Ref. \\
\hline \multicolumn{5}{|l|}{ Spectral type: } \\
\hline Of companion . & $\mathrm{T}$ dwarf & 1 & $\mathrm{~T}$ dwarf & 9 \\
\hline Of primary ................. & M1 V & 2 & $\mathrm{~K} 4 \mathrm{~V}+\mathrm{M} 1.5 \mathrm{~V}+\mathrm{M} 3 \mathrm{~V}$ & 9 \\
\hline$J \quad \ldots \ldots \ldots \ldots \ldots \ldots \ldots \ldots \ldots$ & $14.2 \pm 0.1$ & 3 & $15.33 \pm 0.05$ & 9 \\
\hline$H \quad \ldots \ldots \ldots \ldots \ldots \ldots \ldots \ldots \ldots$ & $14.3 \pm 0.1$ & 3 & $15.28 \pm 0.09$ & 9 \\
\hline$K_{s} \ldots \ldots \ldots \ldots \ldots \ldots \ldots \ldots \ldots \ldots$ & $14.3 \pm 0.1$ & 3 & $15.27 \pm 0.17$ & 9 \\
\hline$J-H \ldots \ldots \ldots \ldots \ldots \ldots \ldots \ldots$ & $-0.1 \pm 0.2$ & 3 & $0.05 \pm 0.10$ & 9 \\
\hline$H-K_{s} \ldots \ldots \ldots \ldots \ldots \ldots \ldots \cdots \cdots$ & $0.0 \pm 0.2$ & 3 & $0.01 \pm 0.19$ & 9 \\
\hline$J-K_{s} \ldots \ldots \ldots \ldots \ldots \ldots \ldots \ldots \ldots$ & $-0.1 \pm 0.2$ & 3 & $0.06 \pm 0.17$ & 9 \\
\hline$\rho(\operatorname{arcsec}) \ldots \ldots \ldots \ldots \ldots \ldots \ldots$ & 7.7 & 4 & $2 \overline{58} 8^{a}$ & 9 \\
\hline 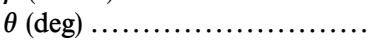 & 164 & 4 & $316^{\mathrm{a}}$ & 9 \\
\hline$\pi_{\text {trig }}(\operatorname{arcsec}) \ldots \ldots \ldots \ldots \ldots \ldots \ldots$ & $0.1732 \pm 0.0011$ & 5 & $0.1693 \pm 0.0017$ & 5 \\
\hline Physical separation (AU)...... & $\sim 45$ & - & $\sim 1500^{\mathrm{a}}$ & - \\
\hline 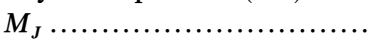 & $15.4 \pm 0.1$ & 3 & $16.47 \pm 0.05$ & 9 \\
\hline$M_{H} \ldots \ldots \ldots \ldots \ldots \ldots \ldots \ldots \ldots \ldots$ & $15.5 \pm 0.1$ & 3 & $16.42 \pm 0.09$ & 9 \\
\hline 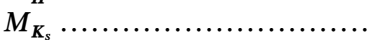 & $15.5 \pm 0.1$ & 3 & $16.41 \pm 0.17$ & 9 \\
\hline Estimated age $(\mathrm{Gyr}) \ldots \ldots \ldots \ldots$ & $0 . \overline{6}-5$ & $6,7,8$ & $2-10$ & 9 \\
\hline Estimated mass $\left(M_{\odot}\right) \ldots \ldots \ldots$ & $0.035 \pm 0.015$ & $6,7,8$ & $0.050 \pm 0.020$ & 9 \\
\hline
\end{tabular}

Notes.-Physical association of the companion with the primary was established via common proper motion by Nakajima et al. 1995 for G1 229B and Burgasser et al. 2000 for G1 570D.

${ }^{a}$ Measured with respect to the A component.

REFERENCES.- (1) Oppenheimer et al. 1995 and Kirkpatrick et al. 1999b; (2) Boeshaar 1976 and Kirkpatrick et al. 1991; (3) Matthews et al. 1996; (4) Golimowski et al. 1998; (5) Perryman et al. 1997; (6) Nakajima et al. 1995; (7) Marley et al. 1996; (8) Leggett et al. 1999; (9) Burgasser et al. 2000.

Theoretical cooling tracks suggest that it has a mass of $0.035 \pm 0.015 M_{\odot}$. The L8 dwarf G1 $584 \mathrm{C}$ has $M_{J}=15.0$, just 0.4 mag brighter in $M_{J}$ than the well known T dwarf Gl 229B. Gl $584 \mathrm{C}$ is between 1.0 and $2.5 \mathrm{Gyr}$ old, has $[\mathrm{Fe} /$ $\mathrm{H}]=-0.20$, and has an inferred mass of $0.060 \pm 0.015$ $M_{\odot}$. Both L dwarfs lie over 1000 AU away from their primaries.

In a related paper, Gizis et al. (2001a) conclude that the dearth of brown dwarf companions - seen at separations less than $3 \mathrm{AU}$ from main-sequence stars - does not continue at very wide separations. This conclusion is based on the two 2MASS discoveries above and on the 2MASS discovery of the T dwarf companion Gl 570D (Burgasser et al. 2000). Each of these companions was found serendipitously during the course of field searches for $\mathrm{L}$ and $\mathrm{T}$ dwarfs, and the statistics for such widely separated brown dwarf companions is still poor. A dedicated 2MASS search specifically looking for companions to nearby stars is currently underway in an effort to bolster the statistics. First results from this survey will be presented in Wilson et al. (2001).

J. D. K. acknowledges the support of the Jet Propulsion Laboratory, California Institute of Technology, which is operated under contract with the National Aeronautics and Space Administration. We thank Joel Aycock, Wayne Wack, and Barbara Schaefer for the outstanding job they did in obtaining the Keck LRIS service observations on
1999 March 31; Alice Monet and Steve Levine for obtaining some of the astrometric data (Table 1) used for common proper motion verification; John Stauffer and Dave Soderblom for enlightening discussions; and Lynne Hillenbrand and John Carpenter for acquiring data in 2000 December used in the telluric absorption discussion of $\S 3.3$. I. N. R. and J. L. acknowledge funding through a NASA/JPL grant to 2MASS Core Project science. The finder charts of Figure 1 make use of the Digitized Sky Survey, which was produced at the Space Telescope Science Institute under US Government grant NAGW-2166. Research in this paper has also relied partly on photographic plates obtained at the Palomar Observatory 48 inch Oschin Telescope for the Second Palomar Observatory Sky Survey which was funded by the Eastman Kodak Company, the National Geographic Society, the Samuel Oschin Foundation, the Alfred Sloan Foundation, the National Science Foundation grants AST84-08225, AST87-19465, AST90-23115, and AST93-18984, and the National Aeronautics and Space Administration grants NGL 05002140 and NAGW 1710. This research has also made use of the SIMBAD database, operated at CDS, Strasbourg, France. This publication makes use of data from the Two Micron All Sky Survey, which is a joint project of the University of Massachusetts and the Infrared Processing and Analysis Center, funded by the National Aeronautics and Space Administration and the National Science Foundation.
Bailer-Jones, C. A. L., \& Mundt, R. 1999, A\&A, 348, 800 .2001, A\&A, 367, 218

Baliunas, S. L., et al. 1995, ApJ, 438, 269

Basri, G., Mohanty, S., Allard, F., Hauschildt, P. H., Delfosse, X., Martín,

E. L., Forveille, T., \& Goldman, B. 2000, ApJ, 538, 363

Bidelman, W. P. 1951, ApJ, 113, 304

Bodenheimer, P. 1965, ApJ, 142, 451

\section{REFERENCES}

Boesgaard, A. M. 1989, ApJ, 336, 798 . 1991, ApJ, 370, L95

Boesgaard, A. M. \& Friel, E. D. 1990, ApJ, 351, 467

Boeshaar, P. C. 1976, Ph.D. thesis, Ohio State Univ.

Burgasser, A. J., et al. 2000, ApJ, 531, L57

Burrows, A., et al. 1997, ApJ, 491, 856

Close, L. M., Richer, H. B., \& Crabtree, D. R. 1990, AJ, 100, 1968 
Donahue, R. A. 1993, Ph.D. thesis, New Mexico State Univ.

. 1998, in ASP Conf. Ser. 154, Cool Stars, Stellar Systems, and the Sun, ed R. A. Donahue \& J. A. Bookbinder (San Francisco: ASP), 1235

Duncan, D. K. 1981, ApJ, 248, 651

Duncan, D. K., Baliunas, S. L., Noyes, R. W., Vaughan, A. H., Frazer, J., \& Lanning, H. H. 1984, PASP, 96, 707

Edvardsson, B., Andersen, J., Gustafsson, B., Lambert, D. L., Nissen, P. E., \& Tomkin, J. 1993, A\&A, 275, 101

Edwards, T. W. 1976, AJ, 81, 245

Eggen, O. J. 1963, AJ, 68, 697

Epchtein, N., et al. 1997, Messenger, 87, 27

Fischer, D. A., \& Marcy, G. W. 1992, ApJ, 396, 178

Friel, E. D., \& Boesgaard, A. M. 1992, ApJ, 387, 170

Gaidos, E. J. 1998, PASP, 110, 1259

Gaidos, E. J., Henry, G. W., \& Henry, S. M. 2000, AJ, 120, 1006

Gizis, J. E., Kirkpatrick, J. D., Burgasser, A., Reid, I. N., Monet, D. G., Liebert, J., \& Wilson, J. C. 2001a, ApJ, 551, L163

Gizis, J. E., Kirkpatrick, J. D., \& Wilson, J. C. 2001 b, AJ, 121, 2185

Gizis, J. E., Monet, D. G., Reid, I. N., Kirkpatrick, J. D., Liebert, J., \& Williams, R. J. 2000, AJ, 120, 1085

Goldman, B., et al. 1999, A\&A, 351, L5

Golimowski, D. A., Burrows, C. J., Kulkarni, S. R., Oppenheimer, B. R., \& Brukardt, R. A. 1998, AJ, 115, 2579

Hale, A. 1994, AJ, 107, 306

Hambly, N. C., Steele, I. A., Hawkins, M. R. S., \& Jameson, R. F. 1995, MNRAS, 273, 505

Hamuy, M., Suntzeff, N. B., Heathcote, S. R., Walker, A. R., Gigoux, P., \& Phillips, M. M. 1994, PASP, 106, 566

Hempelmann, A., Schmitt, J. H. M. M., Schultz, M., Rüdiger, G., \& Stepien, K. 1995, A\&A, 294, 515

Henry, T. J., Soderblom, D. R., Donahue, R. A., \& Baliunas, S. L. 1996, AJ, 111,439

Herbig, G. H. 1965, ApJ, 141, 588

Jeffries, R. D. 1995, MNRAS, 273, 559

Johnson, D. R. H., \& Soderblom, D. R. 1987, AJ, 93, 864

Kirkpatrick, J. D., Allard, F., Bida, T., Zuckerman, B., Becklin, E. E., Chabrier, G., \& Baraffe, I. 1999a, ApJ, 519, 834

Kirkpatrick, J. D., Henry, T. J., \& McCarthy, D. W., Jr. 1991, ApJS, 77, 417

Kirkpatrick, J. D., et al. 1999b, ApJ, 519, 802

Kirkpatrick, J. D., et al. 2000, AJ, 120, 447

Krishnamurthi, A., et al. 1998, ApJ, 493, 914

Lachaume, R., Dominik, C., Lanz, T., \& Habing, H. J. 1999, A\&A, 348, 897

Latham, D. W., Schechter, P., Tonry, J., Bahcall, J. N., \& Soneira, R. M. 1984, ApJ, 281, L41

Leggett, S. K. 1992, ApJS, 82, 351

Leggett, S. K., Toomey, D. W., Geballe, T. R., \& Brown, R. H. 1999, ApJ, 517, L139

Lowrance, P. J., et al. 1999, ApJ, 512, L69

Lowrance, P. J., et al. 2000, ApJ, 541, 390

Maggio, A., Sciortino, S., Vaiana, G. S., Major, P., Bookbinder, J., Golub, L., Harnden, F. R., \& Rosner, R. 1987, ApJ, 315, 687

Magazzù, A., Martín, E. L., \& Rebolo, R. 1993, ApJ, 404, L17

Marcy, G. W., \& Butler, R. P. 2000, PASP, 112, 137

Marley, M. S., Saumon, D., Guillot, T., Freedman, R. S., Hubbard, W. B., Burrows, A., \& Lunine, J. I. 1996, Science, 272, 1919

Martín, E. L., Brandner, W., \& Basri, G. 1999, Science, 283, 1718

Mason, B. D., Douglass, G., G., \& Hartkopf, W. I. 1999, AJ, 117, 1023

Matthews, K., Nakajima, T., Kulkarni, S. R., \& Oppenheimer, B. R. 1996, AJ, 112, 1678
McCook, G. P., \& Sion, E. M. 1987, ApJS, 65, 603

Micela, G., et al. 1999, A\&A, 341, 751

Monet, D. G., Dahn, C. C., Vrba, F. J., Harris, H. C., Pier, J. R., Luginbuhl, C. B., \& Ables, H. D. 1992, AJ, 103, 638

Müller, E. A., Peytremann, E., \& de la Reza, R. 1975, Sol. Phys., 41, 53

Nakajima, T., Oppenheimer, B. R., Kulkarni, S. R., Golimowski, D. A., Matthews, K., \& Durrance, S. T. 1995, Nature, 378, 463

Nakajima, T., et al. 2000, PASJ, 52, 87

Oke, J. B., et al. 1995, PASP, 107, 375

Oppenheimer, B. R., Kulkarni, S. R., Matthews, K., \& Nakajima, T. 1995, Science, 270, 1478

Perryman, M. A. C., et al. 1997, A\&A, 323, L49

. 1998, A\&A, 331, 81

Rebolo, R., Martín, E. L., \& Magazzù, A. 1992, ApJ, 389, L83

Rebolo, R., Zapatero Osorio, M. R., Madruga, S., Béjar, V. J. S., Arribas, S., \& Licandro, J. 1998, Science, 282, L113

Reeves, H., \& Meyer, J.-P. 1978, ApJ, 226, 613

Reid, I. N. 1999, in Proceedings of Star Formation 1999, ed. T. Nakamoto (Nobeyama: Nobeyama Radio Obs.), 327

Reid, I. N., et al. 1999, ApJ, 521, 613

Reid, I. N., Gizis, J. E., Kirkpatrick, J. D., \& Koerner, D. W. 2001, AJ, 121, 489

Retterer, J. M., \& King, I. R. 1982, ApJ, 254, 214

Schmitt, J. H. M. M., Micela, G., Sciortino, S., Vaiana, G. S., Harnden, F. R., Jr., \& Rosner, R. 1990, ApJ, 351, 492

Schweitzer, A., Gizis, J. E., Hauschildt, P. H., Allard, F., \& Reid, I. N. 2001, ApJ, submitted

Skrutskie, M. F., et al. 1997, in The Impact of Large-Scale Near-IR Sky Surveys, ed. F. Garzon (Dordrecht: Kluwer), 25

Skumanich, A. 1972, ApJ, 171, 565

Soderblom, D. R. 1983, ApJS, 53, 1

Soderblom, D. R., Jones, B. F., Balachandran, S., Stauffer, J. R., Duncan, D. K., Fedele, S. B., \& Hudon, J. D. 1993a, AJ, 106, 1059

Soderblom, D. R., \& Mayor, M. 1993, AJ, 105, 226

Soderblom, D. R., Pilachowski, C. A., Fedele, S. B., \& Jones, B. F. 1993b, AJ, 105, 2299

Stauffer, J. R., Schultz, G., \& Kirkpatrick, J. D. 1998, ApJ, 499, 199

Stauffer, J. R., et al. 1999, ApJ, 527, 219

Stern, R. A., Schmitt, J. H. M. M., \& Kahabka, P. T. 1995, ApJ, 448, 683

Struve, O., \& Franklin, K. 1955, ApJ, 121, 337

Tinney, C. G., \& Tolley, A. J. 1999, MNRAS, 304, 119

Upgren, A. R., Grossenbacher, R., Penhallow, W. S., MacConnell, D. J., \& Frye, R. L. 1972, AJ, 77, 486

van Altena, W. F., Lee, J. T., \& Hoffleit, E. D. 1995, The General Catalogue of Trigonometric Stellar Parallaxes (Fourth ed.; Schenectady: Davis Press)

van Biesbroeck, G. 1944, AJ, 51, 61

. 1961, AJ, 66, 528

Ventura, P., Zeppieri, A., Mazzitelli, I., \& D’Antona, F. 1998, A\&A, 331, 1011

Walter, F. M., \& Barry, D. C. 1991, in The Sun in Time, ed C. P. Sonett, M. S. Giampapa, \& M. S. Matthews (Tucson: Univ. Arizona Press), 633

Weinberg, M. D. 1990, in Baryonic Dark Matter, ed. D. Lynden-Bell \& G. Gilmore (London: Kluwer), 117

Wilson, J. C., et al. 2001, AJ, submitted

York, D. G., et al. 2000, AJ, 120, 1579

Zuckerman, B., \& Becklin, E. E. 1992, ApJ, 386, 260 